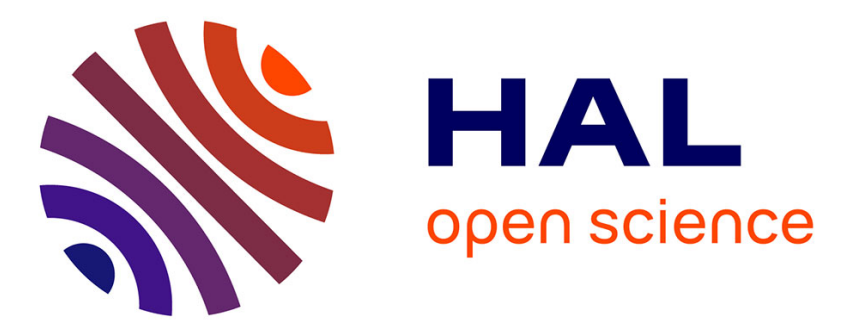

\title{
Three-dimensional FE2 method for the simulation of non-linear, rate-dependent response of composite structures
}

El-Hadi Tikarrouchine, Georges Chatzigeorgiou, Francis Praud, Boris

Piotrowski, Yves Chemisky, Fodil Meraghni

\section{To cite this version:}

El-Hadi Tikarrouchine, Georges Chatzigeorgiou, Francis Praud, Boris Piotrowski, Yves Chemisky, et al.. Three-dimensional FE2 method for the simulation of non-linear, rate-dependent response of composite structures. Composite Structures, 2018, 193, pp.165-179. 10.1016/j.compstruct.2018.03.072 . hal-01762458

\section{HAL Id: hal-01762458 \\ https://hal.science/hal-01762458}

Submitted on 10 Apr 2018

HAL is a multi-disciplinary open access archive for the deposit and dissemination of scientific research documents, whether they are published or not. The documents may come from teaching and research institutions in France or abroad, or from public or private research centers.
L'archive ouverte pluridisciplinaire HAL, est destinée au dépôt et à la diffusion de documents scientifiques de niveau recherche, publiés ou non, émanant des établissements d'enseignement et de recherche français ou étrangers, des laboratoires publics ou privés. 


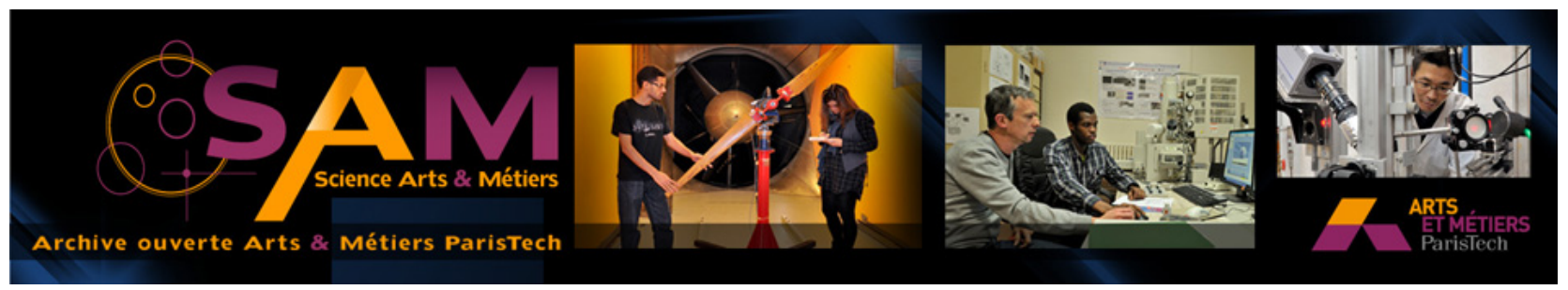

\section{Science Arts \& Métiers (SAM)}

is an open access repository that collects the work of Arts et Métiers ParisTech researchers and makes it freely available over the web where possible.

This is an author-deposited version published in: http://sam.ensam.eu Handle ID: .http://hdl.handle.net/null

\section{To cite this version :}

El-Hadi TIKARROUCHINE, George CHATZIGEORGIOU, Francis PRAUD, Boris PIOTROWSKI, Yves CHEMISKY, Fodil MERAGHNI - Three-dimensional FE2 method for the simulation of nonlinear, rate-dependent response of composite structures - Composite Structures p.in press - 2018 


\title{
Three-dimensional $\mathrm{FE}^{2}$ method for the simulation of non-linear, rate-dependent response of composite structures
}

\author{
E. Tikarrouchine ${ }^{\mathrm{a}, \mathrm{b}}$, G. Chatzigeorgiou ${ }^{\mathrm{a}}$, F. Praud ${ }^{\mathrm{a}}$, B.Piotrowski $^{\mathrm{a}}$, Y. Chemisky ${ }^{\mathrm{a}}$, F. Meraghnia,* \\ ${ }^{a}$ Arts et Metiers ParisTech, LEM3-UMR 7239 CNRS, 4 rue Augustin Fresnel, 57078 Metz, France \\ ${ }^{b}$ Ecole Militaire Polytechnique (EMP) BP17, Bordj El-Bahri, 16111 Alger, Algérie
}

\begin{abstract}
In this paper, a two scale Finite Element method $\left(\mathrm{FE}^{2}\right)$, is presented to predict the non-linear macroscopic response of 3D composite structures with periodic microstructure that exhibit a timedependent response. The sensitivity to the strain rate requires an homogenization scheme to bridge the scales between the macroscopic boundary conditions applied and the local evaluation of the strain rate. In the present work, the effective response of composite materials where the matrix has a local elasto-viscoplastic behavior with ductile damage are analyzed using periodic homogenization, solving simultaneously finite element problems at the microscopic scale (unit cell) and at the macroscopic scale. This approach can integrate any kind of periodic microstructure with any type of non-linear behavior for the constituents (without the consideration of non-linear geometric effects), allowing to treat complex mechanisms that can occur in every phase and at their interface. The numerical implementation of this simulation strategy has been performed with a parallel computational technique in ABAQUS/Standard,with the implementation of a set of dedicated scripts. The homogenization process is performed using a user-defined constitutive law that solve a set full-field non-linear simulations of a Unit Cell and perform the necessary homogenization of the mechanical quantities. The effectiveness of the method is demonstrated with three examples of $3 \mathrm{D}$ composite structures with plastic or viscoplastic and ductile damage matrix. In the first example, the numerical results obtained by this full field approach are compared with a semi-analytical solution on elastoplastic multilayer composite structure. The second example investigates the macroscopic response of a complex viscoplastic composite structure with ductile damage and is compared with the mean field Mori-Tanaka method. Finally, 3D corner structure
\end{abstract}


consisting of periodically aligned short fibres composite is analysed under complex loading path. These numerical simulations illustrate the capabilities of the $\mathrm{FE}^{2}$ strategy under non-linear regime. when time dependent constitutive models describe the response of the constituents Keywords: Multi-scale finite element computation, $\mathrm{FE}^{2}$ method, periodic homogenization, composite materials, elastoviscoplastic behavior, ductile damage. 


\section{Introduction}

Polymer based composite materials are considered to be a good technological solution for automotive and aeronautic industries, thanks to their structural durability and their lightness. A major preoccupation of these industries is to predict the response of such structures with in-service loadings. This requires the development of predictive models that are able to capture the microstructure impact on the mechanical response, and the proper identification of the mechanical properties of the constituents. In this purpose, advanced modelling and simulation methods that integrate the effect of the microstructure is an active area of research. According to the bibliography, several numerical approaches have been proposed for the numerical simulation of the non-linear response of polymer based composite structures including:

i) Phenomenological models, which predict the overall response of the composite materials without taking into account the effect of the different constituents observed at the microscopic scale. Several authors have proposed constitutive models that integrate various rheologies and deformation mechanisms, i.e. viscoelasticity [1, 2], viscoplasticity [3, 4, 5], coupled viscoelasticity and viscoplasticity [6, 7], or even both coupled viscoelasticity, viscoplasticity and damage $[8,9,10,11]$;

ii) Multi-scale methods, that can be classified into two main categories: mean-field and full field approaches. The mean-field approaches are used to describe the behavior of composites for certain categories of microstructures through the Mori-Tanaka Method [12, 13] or the self-consistent scheme [14, 15, 16, 17]. These methodologies have been developed in order to estimate the overall behavior of the composite using average stress and strain quantities for each material phase [18, 19, 20]. These methods have been proved to be accurate for the linear cases. However, for non-linear constitutive laws, especially when the matrix phase exhibits a non-linear behavior, the response of these approaches is inaccurate. It is commonly observed in the literature that the response of the composite obtained by mean-field methods appears to be stiffer than the reality especially when the matrix is ductile and the reinforcements are stiffer [21, 22, 23]. The numerical simulation of these composite systems has necessitated the development of full-field approaches. To determine the response of a 
composite structure, accounting for the description of the microstructure, the so-called $\mathrm{FE}^{2}$ method, appear to be an adequate solution. The major benefit of the $\mathrm{FE}^{2}$ method is the ability to analyse complex mechanical problems with heterogeneous phases that present a variety of behavior at different scales. This idea was originally introduced by Feyel [24], then this method was used and developed by several authors, for example [25, 26, 27, 28, 29, 30, 31]. The majority of these works consider two-dimensional structures, which if they provide a good study case for the analysis of the capabilities of the method, is of limited interest for practical use for the prediction of the overall response of heterogeneous materials and composites, since the spatial arrangement of the phases is mostly three-dimensional.

In this paper, a two-level $\mathrm{FE}^{2}$ method, based on the concept of periodic homogenization under the small strain assumption is implemented in a commercial FE code (ABAQUS/Standard). The method predicts the 3D non-linear macroscopic behavior of a composite with periodic microstructure by considering that each macroscopic integration point is a material point where the characteristics at the macroscopic scale are represented by its own unit cell, which includes the material and geometrical characteristics of the constituents (fibre, matrix) in the microstructure. Therefore, a multilevel finite element analysis has been developed using an implicit resolution scheme, with the use of a Newton-Raphson algorithm to solve simultaneously the non-linear system of equations on the two scales (macroscopic and microscopic).

The main advantage of this methodology is that it can account for any type of non-linear behavior of the constituents (plasticity, viscoelasticity, viscoplasticity and damage), as well as any type of periodic microstructure. The proposed $\mathrm{FE}^{2}$ approach is implemented through a parallelization technique, leading to a significant reduction of the computational time.

The layout of this paper is as follows: in section 2, the theoretical formulation of the homogenization theory is described as well as the principle of scale transition between the local and the global fields. The section also presents the rate dependent constitutive law considered for the matrix phase. In section 3, details of the numerical implementation of the $\mathrm{FE}^{2}$ method is given for a 3D non-linear problem in ABAQUS/Standard with the parallel implementation. In section 4, the approach is validated by comparing the $\mathrm{FE}^{2}$ results with semi-analytical method on 3D multilayer 
composite structure. Afterwards, an example of 3D composite structure exhibiting non-uniform strain fields, in which the microstructure consists of an elastoviscoplastic polymer matrix with ductile damage, reinforced by short glass fibres is presented. The numerical results of the simulation are compared with the Mori-Tanaka method. Finally, the capabilities of this method are shown by simulating the mechanical response of a more complex structure under complex loading path with different strain rate.

\section{Theoretical background and Scale transition}

In this section, the periodic homogenization principle, as well as the transition between the two scales (microscopic and macroscopic) are presented. The principal objective is to determine the macroscopic quantities (stress and tangent modulus) that are obtained through periodic homogenization by accounting for the different mechanisms that exist in the microscopic level, as non-linear plastic/viscoplastic behavior with ductile damage of the matrix. After that, the local constitutive law of each constituent is presented, where a linear elastic law is chosen for the reinforcement and a constitutive model that incorporate elastoviscoplasticity coupled with ductile damage for the matrix.

\subsection{Theoretical background for periodic homogenization}

The objective of the periodic homogenization theory is to define a fictitious homogenized medium having an equivalent response of the heterogeneous medium that is representative of the microstructure. A periodic medium is characterized by a repeated unit cell in the three spatial directions, which forms an unit cell. The theory of periodic homogenization is valid as long as the separation between the scales exists, i.e. the sizes of the unit cell are much smaller than the macroscopic sizes of the medium $(\overline{\boldsymbol{x}}>>\boldsymbol{x})$ (Fig.1). In this paper, the notation $(\bar{\bullet})$ will be used to denote macroscopic quantities. The motion of any macroscopic and microscopic material points $\bar{M}(\bar{x})$ and $M(\bar{x}, x)$ respectively, are governed by the macroscopic and the microscopic equations (Tab,1).

In Tab. $1 \sigma, \varepsilon, \bar{\sigma}$ and $\bar{\varepsilon}$ represent the microscopic and the macroscopic stress and strain tensors respectively, $\overline{\boldsymbol{b}}_{v}$ is the body forces, $V$ and $\bar{V}$ are the volumes of the micro and the macro structure. 


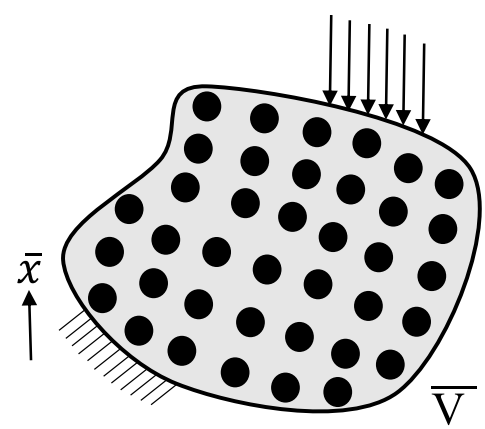

Heterogeneous medium (macro-scale)

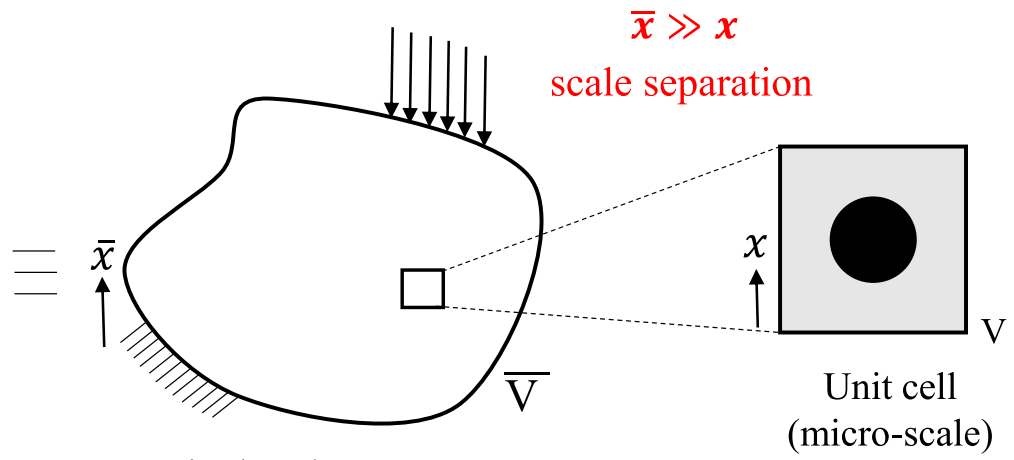

Equivalent homogeneous medium (macro-scale)

Figure 1: Schematic representation of the homogenization computational.

Table 1: Macroscopic and microscopic scale transition [32]

\begin{tabular}{ccc}
\hline Equations & Macro-scale & Micro-scale \\
& $\forall \overline{\boldsymbol{x}} \in \bar{V}$ & $\forall \overline{\boldsymbol{x}} \in \bar{V}, \forall \boldsymbol{x} \in V$ \\
\hline \hline Equilibrium & $\boldsymbol{\operatorname { d i v }}_{\overline{\boldsymbol{x}}}(\overline{\boldsymbol{\sigma}})+\overline{\boldsymbol{b}}_{v}=0$ & $\boldsymbol{\operatorname { d i v }}_{\boldsymbol{x}}(\boldsymbol{\sigma})=0$ \\
Kinematics & $\overline{\boldsymbol{\varepsilon}}=\frac{1}{2}\left(\boldsymbol{G r a d}_{\bar{x}}(\overline{\boldsymbol{u}})+\mathbf{G r a d}_{\bar{x}}^{\top}(\overline{\boldsymbol{u}})\right)$ & $\boldsymbol{\varepsilon}=\frac{1}{2}\left(\boldsymbol{G r a d}_{x}(\boldsymbol{u})+\mathbf{G r a d}_{x}^{\top}(\boldsymbol{u})\right)$ \\
Constitutive law & $\overline{\boldsymbol{\sigma}}=\overline{\boldsymbol{F}}(\overline{\boldsymbol{x}}, \overline{\boldsymbol{\varepsilon}})$ & $\boldsymbol{\sigma}=\boldsymbol{F}(\overline{\boldsymbol{x}}, \boldsymbol{x}, \boldsymbol{\varepsilon})$ \\
Strain energy rate & $\dot{\bar{W}}_{\varepsilon}=\overline{\boldsymbol{\sigma}}: \dot{\overline{\boldsymbol{\varepsilon}}}$ & $\dot{W}_{\varepsilon}=\boldsymbol{\sigma}: \dot{\boldsymbol{\varepsilon}}$ \\
\hline
\end{tabular}

Moreover, $\boldsymbol{x}, \overline{\boldsymbol{x}}, \boldsymbol{u}$ and $\overline{\boldsymbol{u}}$ are the microscopic and the macroscopic positions and displacement vectors, while $\boldsymbol{F}$ and $\overline{\boldsymbol{F}}$ are operators that define the micro and macro relationships between the stress and strain. Both $\boldsymbol{F}$ and $\overline{\boldsymbol{F}}$ are considered non-linear operators in this work.

The homogenization theory attempts to define the $\overline{\boldsymbol{F}}$ operator, which characterizes the macroscopic behavior, from the local behaviors defined by the $\boldsymbol{F}$ operator. In order to make this possible, it is necessary to introduce the concept of scale transition between the macro and the micro scales. According to the average stress and strain theorems, it can be demonstrated that the stress and strain averages within the unit cell are equal to the stress and strains corresponding to uniform 
tractions and linear displacements respectively that are applied at its boundaries. These averages represent the macroscopic stress and strain tensors respectively. The relationships between the two scales are given by the following equations:

$$
\begin{gathered}
\overline{\boldsymbol{\sigma}}=\langle\boldsymbol{\sigma}\rangle=\frac{1}{V} \int_{V} \boldsymbol{\sigma} d V=\frac{1}{V} \int_{\partial V} \boldsymbol{\sigma} \cdot \boldsymbol{n} \otimes \boldsymbol{x} d S \\
\overline{\boldsymbol{\varepsilon}}=\langle\boldsymbol{\varepsilon}\rangle=\frac{1}{V} \int_{V} \boldsymbol{\varepsilon} d V=\frac{1}{2 V} \int_{\partial V}(\boldsymbol{u} \otimes \boldsymbol{n}+\boldsymbol{n} \otimes \boldsymbol{u}) d S
\end{gathered}
$$

where $\boldsymbol{n}$ is the outgoing normal of the unit cell boundary $\partial V .\langle\bullet\rangle$ is the mean operator and $\otimes$ the dyadic product.

\subsubsection{Non-linear scale transition: incremental approach}

Since the homogenization is based on the separation between the different scales, the connection between these scales (microscopic and macroscopic problems) should be defined in order to be able to predict the overall behavior of the structure.

\section{Microscopic problem}

The periodicity condition implies that, the displacement field $\boldsymbol{u}$ of any material point located in $\boldsymbol{x}$ can be described by an affine part, in which a periodic fluctuation $\boldsymbol{u}^{\prime}$ is added as is presented in Fig. 2:

$$
\boldsymbol{u}(\overline{\boldsymbol{x}}, \boldsymbol{x}, t)=\overline{\boldsymbol{\varepsilon}}(\overline{\boldsymbol{x}}, t) \cdot \boldsymbol{x}+\boldsymbol{u}^{\prime}(\overline{\boldsymbol{x}}, \boldsymbol{x}, t)
$$
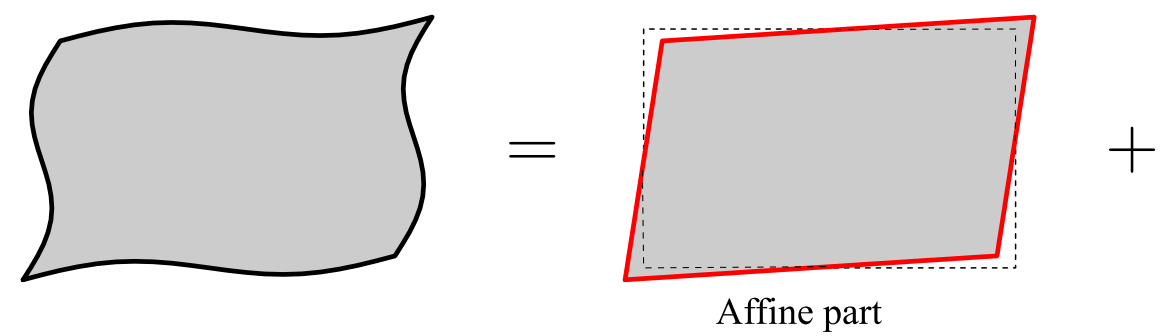

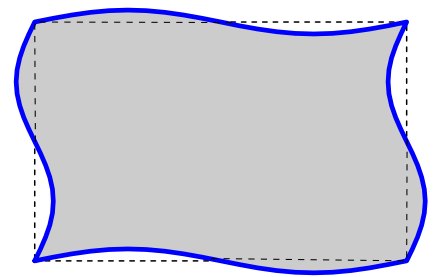

Periodic fluctuation

Figure 2: Definition of the displacement field as the sum of an affine part and a periodic fluctuation 
The periodic fluctuating quantity $\boldsymbol{u}^{\prime}$ takes the same value on each pair of opposite parallel sides of the unit cell and the strain average produced by $\boldsymbol{u}^{\prime}$ is null [Eq. 5]. Therefore, the full strain average is well equal to the macroscopic strain [Eq. 6].

$$
\begin{gathered}
\boldsymbol{\varepsilon}(\boldsymbol{u})=\overline{\boldsymbol{\varepsilon}}+\boldsymbol{\varepsilon}\left(\boldsymbol{u}^{\prime}\right) \\
\left\langle\boldsymbol{\varepsilon}\left(\boldsymbol{u}^{\prime}\right)\right\rangle=\frac{1}{V} \int_{V} \boldsymbol{\varepsilon}\left(\boldsymbol{u}^{\prime}\right) d V=0, \\
\langle\boldsymbol{\varepsilon}(\boldsymbol{u})\rangle=\overline{\boldsymbol{\varepsilon}}+\left\langle\boldsymbol{\varepsilon}\left(\boldsymbol{u}^{\prime}\right)\right\rangle=\overline{\boldsymbol{\varepsilon}}
\end{gathered}
$$

The traction vector $\boldsymbol{\sigma} . \boldsymbol{n}$ is anti periodic and satisfies the conditions of equilibrium within the unit cell. The micro problem is formulated as follows:

$$
\left\{\begin{array}{l}
\sigma=\boldsymbol{F}(\boldsymbol{x}, \boldsymbol{\varepsilon}(\boldsymbol{u}(\boldsymbol{x}))) \quad \forall x \in V, \\
\operatorname{div}_{x}(\sigma(x))=0 \quad \forall x \in V, \\
u_{i}-u_{j}=\bar{\varepsilon} \cdot\left(x_{i}-x_{j}\right) \quad \forall x \in V
\end{array}\right.
$$

where $\boldsymbol{u}_{i}, \boldsymbol{u}_{j}, \boldsymbol{x}_{i}$ and $\boldsymbol{x}_{j}$ are the displacements and the positions of each pair of opposite parallel material point of the unit cell boundary respectively, while $\bar{\varepsilon}$ is the macroscopic strain. The relationship between the microscopic stress and the microscopic strain in incremental approaches is provided by the linearised expression [Eq. 8]:

$$
\Delta \sigma(x)=\mathbb{C}_{t}(x): \Delta \varepsilon(x) \quad \forall x \in V
$$

where $\mathbb{C}_{t}$ is the local tangent operator tensor defined as the numerical differentiation of the stress with respect to the total strain.

\section{Macroscopic problem}

The relationship between macroscopic stress and strain cannot be explicitly provided by a stiffness tensor. Nevertheless, for a given macroscopic strain, the macroscopic stress response can be computed using an implicit resolution scheme, where the local behavior is linearized and corrected at each strain increment [Eq. 8]. Then, using the same incremental methodology, the macroscopic 
behavior can also be linearized in order to predict the next increment. This linearization requires to write the macroscopic constitutive law in the non-linear form of Eq. 9. The equilibrium at the macroscopic level in the absence of body forces is given by the Eq. 10 .

$$
\begin{gathered}
\Delta \overline{\boldsymbol{\sigma}}(\bar{x})=\left\langle\mathbb{C}_{t}(\boldsymbol{x}): \Delta \varepsilon(x)\right\rangle \quad \forall x \in V, \\
\operatorname{div}_{x}(\Delta \overline{\boldsymbol{\sigma}}(\bar{x}))=0 \quad \forall x \in V,
\end{gathered}
$$

where $\Delta \overline{\boldsymbol{\sigma}}(\overline{\boldsymbol{x}})$ is the macroscopic stress tensor associated with the point $\overline{\boldsymbol{x}}$ of the macrostructure at each macroscopic strain increment.

The relationship between the macroscopic stress and strain is given in Voigt notation in Eq. 11 , The macroscopic tangent operator $\overline{\mathbb{C}}_{t}$ is recovered by computing the macroscopic stress resulting from the six elementary strain states written in Eq. 12 (also in Voigt notation) at each macroscopic strain increment:

$$
\begin{aligned}
& \left\{\begin{array}{c}
\Delta \bar{\sigma}_{1} \\
\Delta \bar{\sigma}_{2} \\
\Delta \bar{\sigma}_{3} \\
\Delta \bar{\sigma}_{4} \\
\Delta \bar{\sigma}_{5} \\
\Delta \bar{\sigma}_{6}
\end{array}\right\}=\left(\begin{array}{llllll}
\bar{C}_{t, 11} & \bar{C}_{t, 12} & \bar{C}_{t, 13} & \bar{C}_{t, 14} & \bar{C}_{t, 15} & \bar{C}_{t, 16} \\
& \bar{C}_{t, 22} & \bar{C}_{t, 23} & \bar{C}_{t, 24} & \bar{C}_{t, 25} & \bar{C}_{t, 26} \\
& & \bar{C}_{t, 33} & \bar{C}_{t, 34} & \bar{C}_{t, 35} & \bar{C}_{t, 36} \\
& & & \bar{C}_{t, 44} & \bar{C}_{t, 45} & \bar{C}_{t, 46} \\
& & & & \bar{C}_{t, 55} & \bar{C}_{t, 56} \\
& & & & & \bar{C}_{t, 66}
\end{array}\right\} \times\left\{\begin{array}{c}
\Delta \bar{\varepsilon}_{1} \\
\Delta \bar{\varepsilon}_{2} \\
\Delta \bar{\varepsilon}_{3} \\
2 \Delta \bar{\varepsilon}_{4} \\
2 \Delta \bar{\varepsilon}_{5} \\
2 \Delta \bar{\varepsilon}_{6}
\end{array}\right\}
\end{aligned}
$$

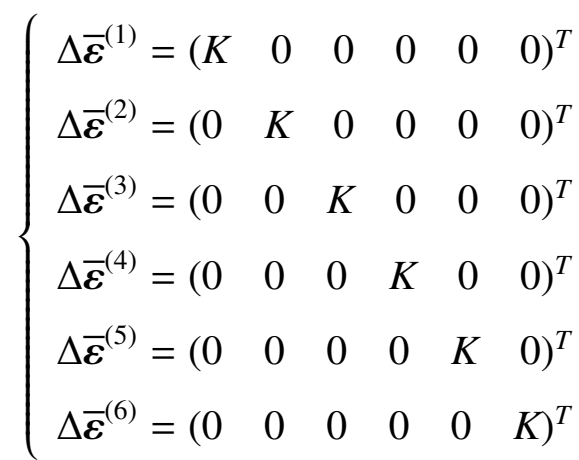

Then, the $i j$ component of the tangent operator is given by the $i^{t h}$ component of the stress vector calculated with the $j^{\text {th }}$ elementary strain state, divided by the $j^{\text {th }}$ component of the strain vector of 
the elementary strain state:

$$
\bar{C}_{t, i j}=\frac{\Delta \overline{\boldsymbol{\sigma}}_{i}^{(j)}}{K}, \quad i, j=1,2,3,4,5,6
$$

Usually, $K$ is chosen to be equal to 1 .

\subsection{Local elasto-viscoplastic behavior with ductile damage for the matrix}

The constitutive law of the matrix material is defined through a thermodynamically based phenomenological model for viscoplasticity and ductile damage in semi-crystalline polymers [33, 34, 11]. These materials exhibit a dissipative behavior that combines solid and fluid properties with some apparent stiffness reduction. The model is described by the rheological scheme given in the Fig. 3. It is composed of: one single linear spring, subjected to an elastic strain $\boldsymbol{\varepsilon}_{e}$, and a viscoplastic branch, subjected to a viscoplastic strain $\boldsymbol{\varepsilon}_{p}$ which consists of a frictional element, a non-linear spring and a non-linear dash-pot. The linear spring and the viscoplastic branch are positioned in series.

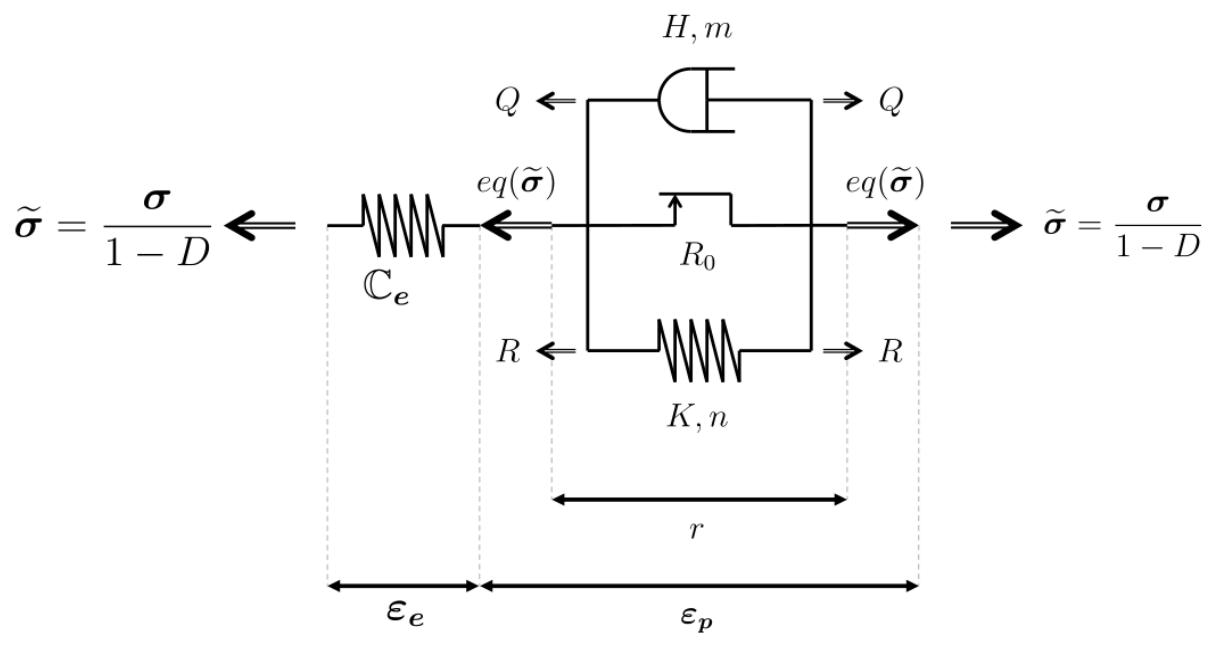

Figure 3: Rheological scheme of the viscoplastic behavior and ductile damage [11].

The model is formulated within the thermodynamics framework [33, 11]. The state laws are obtained by differentiation of the Helmholtz potential with respect to the state variables. This 
potential is formulated as the sum of the stored energies of the spring and the viscoplastic branch.

$$
\rho \psi\left(\boldsymbol{\varepsilon}, r, \boldsymbol{\varepsilon}_{p}, D\right)=\frac{1}{2}\left(\boldsymbol{\varepsilon}-\boldsymbol{\varepsilon}_{p}\right):(1-D) \mathbb{C}_{e}:\left(\boldsymbol{\varepsilon}-\boldsymbol{\varepsilon}_{p}\right)+\int_{0}^{r} R(\xi) \mathrm{d} \xi
$$

The internal state variables $\boldsymbol{\varepsilon}_{p}, r$ and $D$ represent the viscoplastic strain, effective equivalent viscoplastic strain variable and the damage variable respectively. $\mathbb{C}_{e}$ is the initial fourth order stiffness tensor of the single spring, classically defined for bulk isotropic materials. $R$ is the hardening function, chosen under the form of the power law function, that must be increasing, positive and vanishes at $r=0$ :

$$
R(r)=K r^{n}
$$

where $K$ and $n$ are the viscoplastic material parameters. According to the second principle of thermodynamics, dissipation is always positive or null (Clausius Duhem inequality). Assuming that the mechanical and thermal dissipations are uncoupled, the rate of the mechanical dissipated energy $\dot{\Phi}$ is positive or zero and is given by the difference between the strain energy rate $\dot{W}_{\varepsilon}$ and the stored energy rate $\rho \dot{\psi}$ (Eq. 16 ).

$$
\begin{aligned}
\dot{\Phi} & =\dot{W}_{\varepsilon}-\rho \dot{\psi} \\
& =\sigma: \dot{\varepsilon}-\rho\left(\frac{\partial \psi}{\partial \varepsilon}: \dot{\varepsilon}+\frac{\partial \psi}{\partial \varepsilon_{p}}: \dot{\varepsilon}_{p}+\frac{\partial \psi}{\partial r}: \dot{r}+\frac{\partial \psi}{\partial D}: \dot{D}\right) \\
& =\sigma: \dot{\varepsilon}_{p}-R \dot{r}+Y \dot{D} \geq 0 .
\end{aligned}
$$

The viscoplasticity and damage are considered to be coupled phenomena [35, 34]. Consequently, the evolution of $\varepsilon_{p}, r$ and $D$ are described by the normality of a convex indicative function that satisfies the above inequality:

$$
F(\sigma, R, Y ; D)=\underbrace{\frac{\mathrm{eq}(\sigma)}{1-D}-R-R_{0}}_{f(\sigma, R ; D)}+\underbrace{\frac{S}{(\beta+1)(1-D)}\left(\frac{Y}{S}\right)^{\beta+1}}_{f_{D}(Y ; D)}
$$

In the last expression, the term $f(\sigma, R ; D)$ denotes the yield criterion function which activates the mechanism ( $\dot{r}>0$ if $f>0$, else $\dot{r}=0$ ). The function $f$ is expressed in the effective stress space. $f_{D}$ is an additive term that takes into account the evolution of the damage at the same time as the viscoplasticity. eq $(\sigma)$ denotes the von Mises equivalent stress, $R_{0}$ denotes the yield 
threshold, while $S$ and $\beta$ are damage related material parameters. The viscous effect is introduced by considering a relation between the positive part of $f$ and $\dot{r}$ through a function $Q$. This function is chosen under the form of the power law:

$$
\langle f\rangle_{+}=Q(\dot{r}), \quad Q(\dot{r})=H \dot{r}^{m}
$$

where $H$ and $m$ are the material parameters. The function $Q(\dot{r})$ must be increasing, positive and null at $\dot{r}=0$.

This type of model allows to capture some well known effects of thermoplastic polymers, namely the rate effect through the creep and relaxation phenomena, as well as the stiffness reduction due to the ductile damage. Tab. 2 summarizes the thermodynamic variables, the evolution laws and the von Mises type viscoplastic criterion of the model. In the table, $\operatorname{Dev}(\sigma)$ denotes the deviatoric part of the stress.

Observable state variable Associated variable

$$
\varepsilon
$$

$$
\sigma=\rho \frac{\partial \psi}{\partial \boldsymbol{\varepsilon}}=(1-D) \mathbb{C}_{e}:\left(\boldsymbol{\varepsilon}-\boldsymbol{\varepsilon}_{p}\right)
$$

State variables

$$
\begin{array}{cc}
r & R=\rho \frac{\partial \psi}{\partial r}=R(r) \\
\boldsymbol{\varepsilon}_{p} & -\sigma=\rho \frac{\partial \psi}{\partial \boldsymbol{\varepsilon}_{p}} \\
D & Y=\rho \frac{\partial \psi}{\partial D}
\end{array}
$$$$
\text { Multiplier }
$$$$
\text { Criterion }
$$

Associated variables

\section{Evolution laws}

$$
\begin{gathered}
\dot{r}=-\frac{\partial F}{\partial R} \dot{\lambda}=\dot{\lambda} \\
\dot{\boldsymbol{\varepsilon}}_{p}=\frac{\partial F}{\partial \sigma} \dot{\lambda}=\frac{3}{2} \frac{\operatorname{Dev}(\sigma)}{\operatorname{eq}(\sigma)} \frac{\dot{r}}{1-D} \\
\dot{D}=\frac{\partial F}{\partial Y} \dot{\lambda}=\left(\frac{Y}{S}\right)^{\beta} \frac{\dot{r}}{1-D}
\end{gathered}
$$

$$
\lambda=r
$$

$$
f(\sigma, R ; D)=\frac{\mathrm{eq}(\sigma)}{1-D}-R-R_{0}
$$$$
\langle f\rangle_{+}=Q(\dot{r})
$$ 


\section{Multi scale FE computation and numerical implementation}

To predict the macroscopic behavior of a composite structure, taking into account the effect of the microstructure, homogenization scheme within the framework of a $\mathrm{FE}^{2}$ is an accurate solution. According to Feyel [24], this approach considers that the macroscopic problem and the microscopic heterogeneous unit cell are solved simultaneously. On the macroscopic scale, the material is assumed as a homogenized medium with non-linear behavior. The macroscopic response is calculated by solving an appropriate periodic boundary value problem at the microscopic level within a homogenization scheme. The important macroscopic information (strain) passes to the unit cell through the constraint drivers. The concept of constraint drivers is explained in the next subsection.

It is pointed out that the response at the macroscopic scale is obtained by the homogenization process and is frequently called "homogenized". The macroscopic fields and tangent moduli depend on the microscopic response at each unit cell. Since the macroscopic strains are heterogeneous in the structure, the homogenized response varies at every macroscopic point, providing a type of spatial heterogeneity.

\subsection{Unit cell computations for periodic homogenization using the concept of constraint drivers}

The method of constraint drivers is a numerical technique which allows to apply any state of macroscopic stress, strain or even mixed stress/strain on a periodic finite element unit cell. More detailed exposition about this concept is given in [36, 37, 38].

In the finite element framework a unit cell for periodic media should be associated with periodic mesh. This means that for each border node, there must be another node at the same relative position on the opposite side of the unit cell. The aim of the constraint drivers in a periodic homogenization approach is to apply a macroscopic strain $\bar{\varepsilon}$ on the unit cell, taking into account the periodic boundary conditions. In practice, a displacement gradient is applied between each pair of opposite parallel border nodes (denoted by the indices $i$ and $j$ ). This gradient is directly related to the macroscopic strain tensor $\overline{\boldsymbol{\varepsilon}}_{i j}$ by the following general kinematic relationship:

$$
u_{i}^{\prime}=u_{j}^{\prime} \quad \Longleftrightarrow \quad u_{i}-u_{j}=\bar{\varepsilon} .\left(x_{i}-x_{j}\right) \quad \forall x \in V
$$


The proposed method introduces the six components of the macroscopic strain tensor as additional degrees of freedom (constraint drivers) that are linked to the mesh of the unit cell using the kinematic equation 19. The displacements of these additional degrees of freedom, noted as $u_{11}^{c d}, u_{22}^{c d}, u_{33}^{c d}, u_{12}^{c d}, u_{13}^{c d}$ and $u_{23}^{c d}$, take the values of each component of the macroscopic strain tensor $\bar{\varepsilon}_{11}, \bar{\varepsilon}_{22}, \bar{\varepsilon}_{33}, 2 \bar{\varepsilon}_{12}, 2 \bar{\varepsilon}_{13}$ and $2 \bar{\varepsilon}_{23}$, respectively. The dual forces of the constrain drivers are noted as $F_{11}^{c d}, F_{22}^{c d}, F_{33}^{c d}, F_{12}^{c d}, F_{13}^{c d}$ and $F_{23}^{c d}$, respectively, and they permit to recover directly the corresponding components of the macroscopic stress tensor (Fig. 4) at the end of the unit cell calculations. Dividing the dual force by the unit cell volume leads to the corresponding macroscopic stresses.

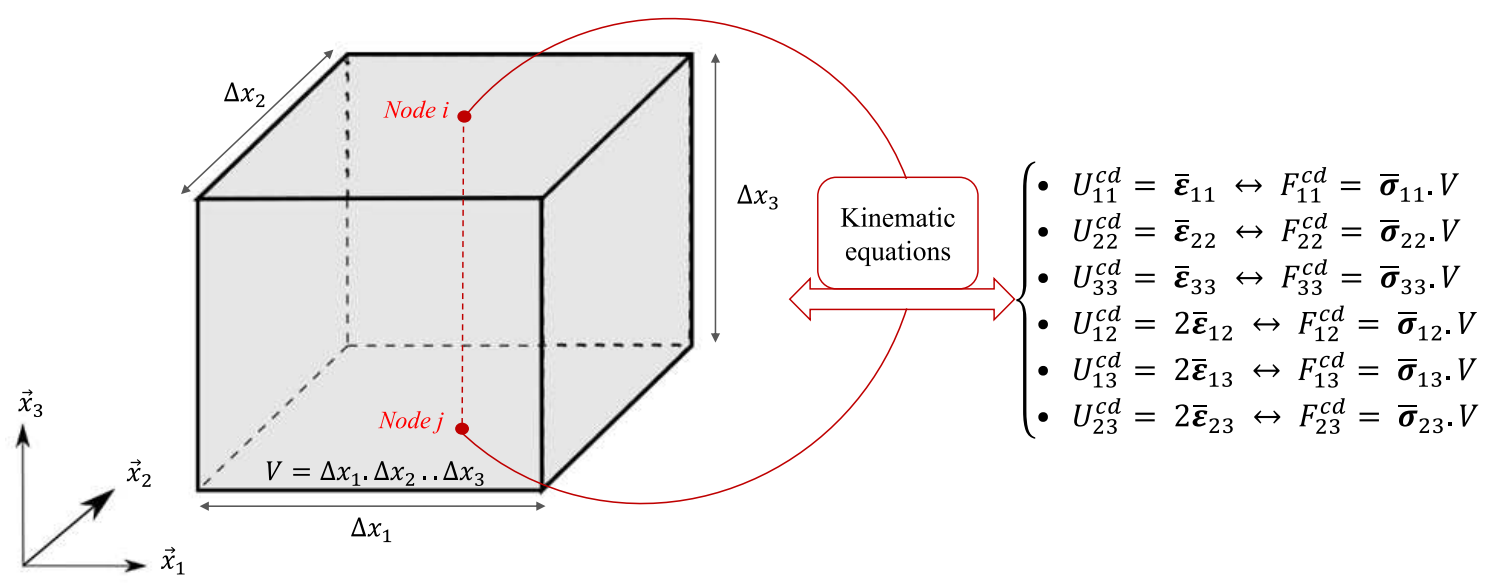

Figure 4: Connection of the constraint drivers with the unit cell.

\subsection{Concept and numerical algorithm of $F E^{2}$ method}

After defining the concept of constraint drivers, the implementation of a two-scale finite element approach is the next step in the computational homogenization framework. The proposed method lies within the general category of multi-scale models. In this method the macroscopic constitutive behavior is calculated directly from the unit cell, providing the geometry and the phenomenological constitutive equations of each constituent. The $\mathrm{FE}^{2}$ method consists of three main steps according to [24]:

(1) A geometrical description and a FE model of the unit cell. 
(2) The local constitutive laws expressing the response of each component of the composite within the unit cell.

(3) Scale transition relationships that define the connection between the microscopic and the macroscopic fields (stress and strain).

The scale transition is provided by the concept of homogenization theory, using volume averaging of microscopic quantities of the unit cell, which is solved thanks to periodic boundary conditions. The macroscopic fields (stress and strain) are introduced in a unit cell by using the six additional degrees of freedom (constraint drivers), that are linked with the boundaries through the kinematic equations [Eq. 19]. The macroscopic behavior of a 3D composite structure is computed by considering that the material response of each macroscopic integration point is established from the homogenization of a unit cell that is connected to each macroscopic integration point. Each unit cell contains the local constitutive laws of different phases and the geometrical characteristics of the microstructure.

The $\mathrm{FE}^{2}$ approach presented here has been developed using an implicit resolution scheme, with the use of a Newton-Raphson algorithm, that solves the non-linear problems at the two scales. At each macroscopic integration point, the macroscopic stress and the macroscopic tangent operator are computed for the calculated macroscopic strain at each time increment, by solving iteratively a FE problem at the microscopic scale.

\subsubsection{Concept of transition between scales in $F E^{2}$ computations}

In the framework of $\mathrm{FE}^{2}$ modelling the global resolution step is performed at each time increment by solving a local equilibrium problem at each macroscopic integration point. At each step, the microscopic problem is solved by applying the macroscopic strain increment to the unit cell through the periodic boundary conditions. The system of equations in the linearized incremental 
form is given as follows:

$$
\left\{\begin{array}{l}
\Delta \sigma(x)=\mathbb{C}_{t}(x): \Delta \varepsilon(x) \quad \forall x \in V, \\
\operatorname{div}_{x}(\Delta \sigma(x))=0 \quad \forall x \in V, \\
\Delta u_{i}-\Delta u_{j}=\Delta \bar{\varepsilon} \cdot\left(x_{i}-x_{j}\right) \quad \forall x \in V
\end{array}\right.
$$

By using the developed user subroutine at the microscopic scale which contains the non-linear local behavior of the constituents, the microscopic stress, tangent operator and internal state variables $V_{k}$ are computed at every microscopic point. The macroscopic stress $\bar{\sigma}$ is then computed through volume averaging of the microscopic stresses, and the local tangent operators of all microscopic points are utilized to obtain the macroscopic tangent operator $\overline{\mathbb{C}}_{t}$ by solving six elastic-type loading cases with the elementary strain states described in subsection 2.1.2. The internal state variables and the local stress are saved as initial conditions for the next time increment. Once the macroscopic quantities $\bar{\sigma}$ and $\overline{\mathbb{C}}_{t}$ are computed, the analysis at the macroscopic level is then performed and the macroscopic strain increment $\Delta \bar{\varepsilon}$ is provided by the Finite Element Analyses Package ABAQUS at every macroscopic point through the global equilibrium resolution. This information is passed to the macroscopic scale by using a user defined constitutive model (denoted here as Meta-UMAT) that represent the behavior of a macroscopic material point and contains the unit cell equations and hence the process returns to the local problem. The iterative procedure inside the Meta-UMAT is depicted in Fig. 5. The loop is repeated until numerical convergence is achieved in both micro and macro-scales numerical problems. After the convergence, the analysis proceeds to the next time step. Both the Meta-UMAT and the structural analysis in the macroscopic level define the $\mathrm{FE}^{2}$ approach.

\subsubsection{Algorithm of $F E^{2}$ and parallel calculation}

The algorithm of the $\mathrm{FE}^{2}$ computational strategy for the non-linear case in ABAQUS/Standard is presented in Fig. 6 .

As shown in Figures 5 and 6 , the macroscopic problem is solved at each increment in a linearized manner, considering the homogenized tangentmod modulus $\overline{\mathbb{C}}_{t}$. The elastic prediction - inelastic correction is performed at the scale of the constituents laws (Micro-UMAT) using the well-known 


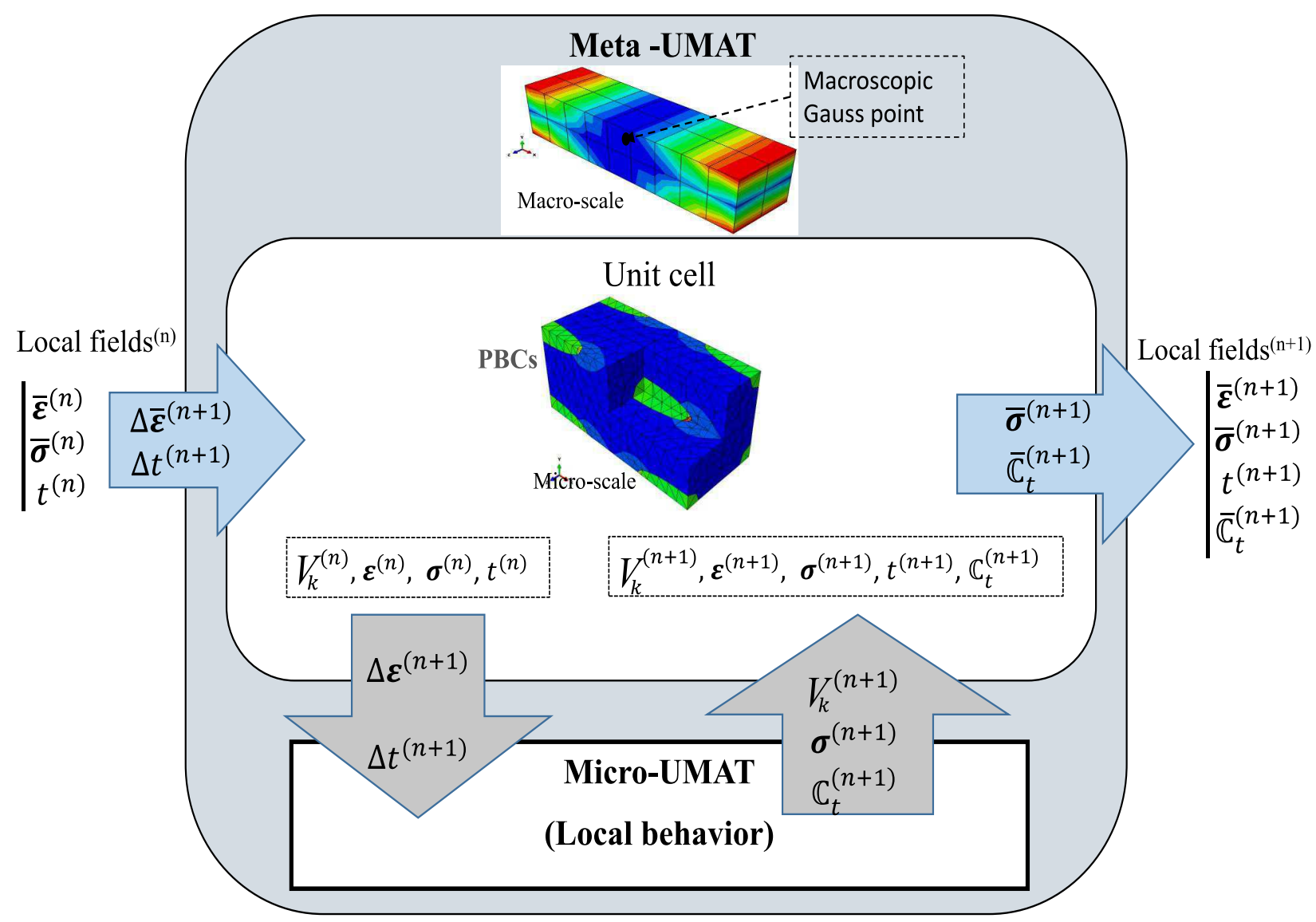

Figure 5: Meta-UMAT for the overall response computation of the composite using $F E^{2}$ approach at time increment $n+1$.

"return mapping algorithm - convex cutting plane" scheme [39].

The aim of the $\mathrm{FE}^{2}$ approach is to perform structural numerical simulations, thus reduction of the computational time is of outmost importance. Since the $\mathrm{FE}^{2}$ homogenization requires very costly computations, parallel calculation procedures for running the analysis on multiple CPUs are unavoidable.

\section{Parallel implementation of the $F E^{2}$ code in ABAQUS/Standard}

It is known that the $\mathrm{FE}^{2}$ computation is expensive in terms of CPU time, caused by the transition between the two scales and the degree of freedom number of the microscopic and the macroscopic models. To reduce this computational time, a parallel implementation of the $\mathrm{FE}^{2}$ procedure is set-up in ABAQUS/Standard. All the Finite Element Analyses of the unit cells within a sin- 


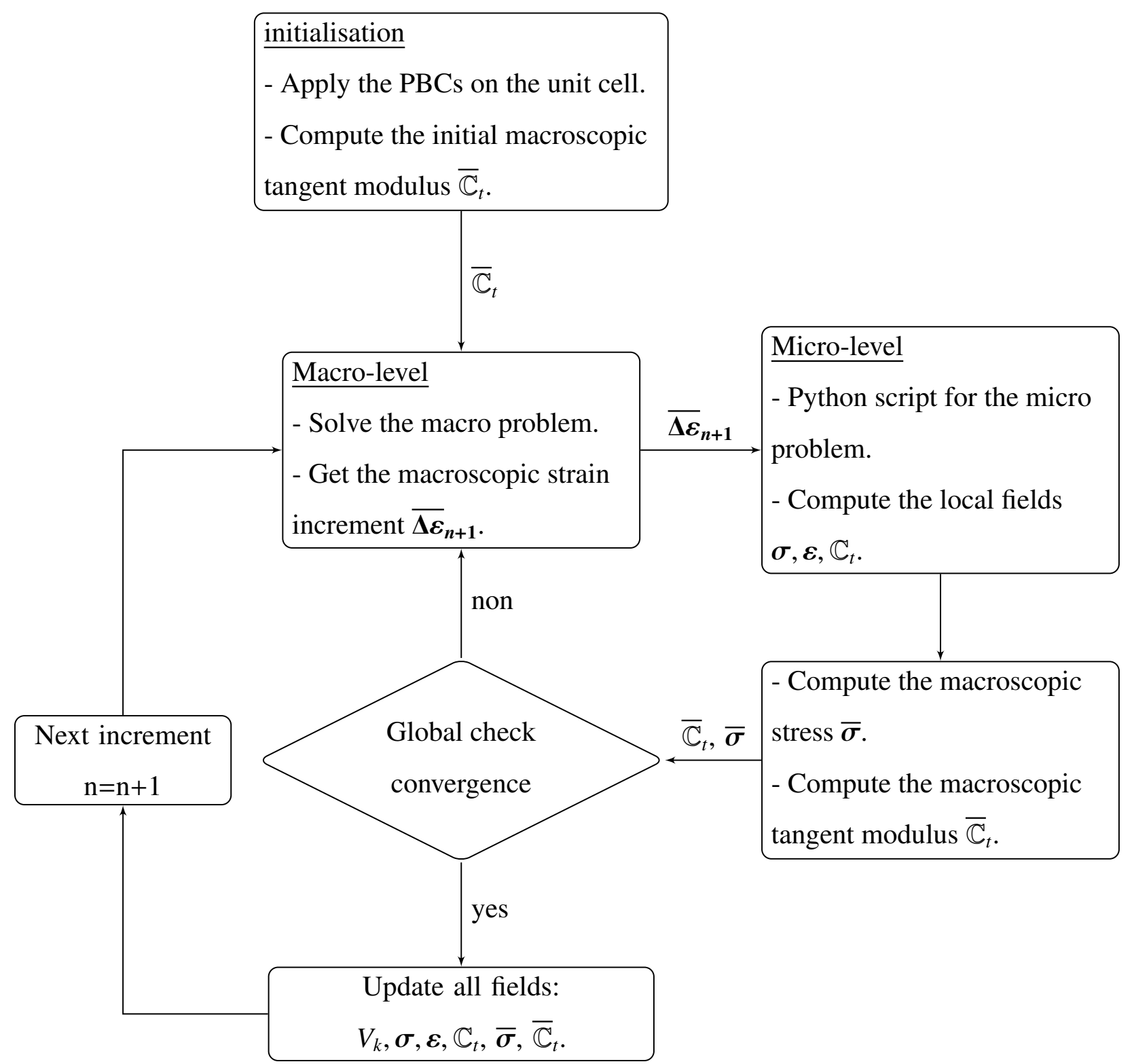

Figure 6: The flow chart of the two scales $\mathrm{FE}^{2}$ algorithm in ABAQUS/Standard for non-linear case.

gle macroscopic element (one per integration point) is sent on a single computation node (a set of processors) and their are solved iteratively. The computations that correspond to each macroscopic element are solved in different computations nodes. Thus, theoretically the parallelization can be performed simultaneously on every element. In practice the parallel computation is limited to the number of available calculation nodes. Note that the computations of every microscopic 
Finite Element Analysis can also be computed in parallel within the computation node if it possess several processors (which is often the case) and this parallelization process is governed by the Finite Element Analyses Package ABAQUS. In practice, the Meta-UMAT calls an appropriate python script that solves the local problem (including the computation of the macroscopic tangent modulus) at each macroscopic integration point, with the use of the microscopic UMAT, which contains the local non-linear behavior of the constituents (Fig. 7). Afterwards, the global solver of ABAQUS checks that all calculations at different processors are completed before proceeding to the resolution of the macroscopic problem, before passing to the next time increment, or the next macroscopic iteration.

\subsection{Implementation of the microscopic problem in ABAQUS}

With regard to the microscopic problem, as mentioned previously, the Meta-UMAT executes a properly designed python script in each macroscopic integration point of the composite structure with the first macroscopic strain increment given by ABAQUS.

The periodic boundary conditions (PBCs) and the macroscopic strain are applied on the unit cell by means of the python script at each time increment, since this last information is given at each integration point from the prediction of the strain increment that should satisfy the global equilibrium. The script also calls the solver ABAQUS to solve the microscopic Finite Element Analyses, which utilize the microscopic user subroutines that contains the local constitutive laws of the constituents, in order to obtain the microscopic response through a return mapping iterative process. Once the local equilibrium is achieved, the local response $\left(\sigma\right.$ and $\left.\mathbb{C}_{t}\right)$ are computed. Then, the macroscopic stress is recovered as a reaction force divided by the unit cell volume on the constraint drivers (section 3.1). The macroscopic tangent modulus is calculated by mapping the local tangent moduli on the unit cell through the six elementary strain states. Through the python script, the macroscopic quantities $\left(\overline{\boldsymbol{\sigma}}\right.$ and $\left.\overline{\mathbb{C}}_{t}\right)$ are calculated and transferred to in the Meta-UMAT. At this point, the global equilibrium is checked, if the convergence is reached, we proceed to the next time increment $n+1$. 


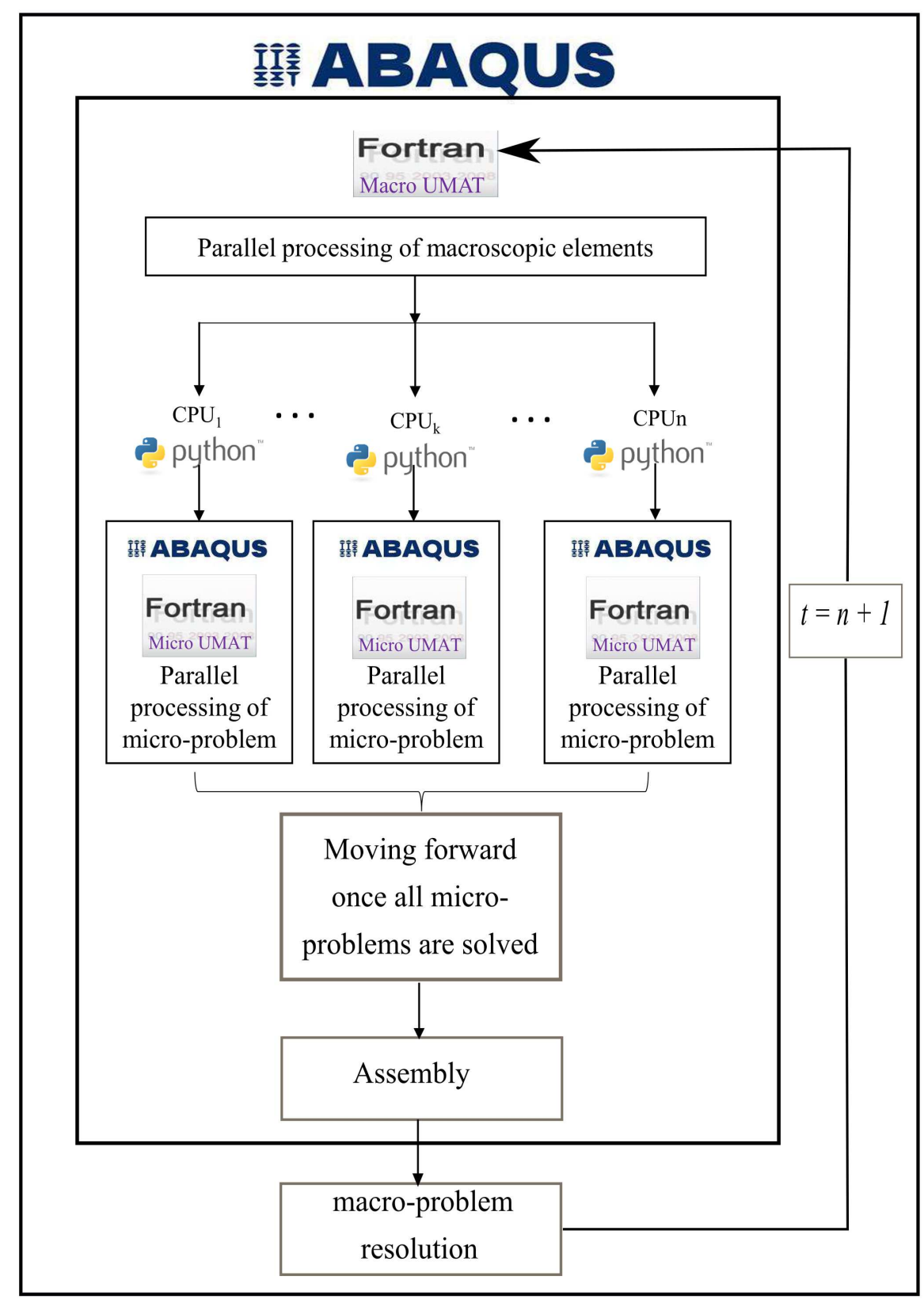

Figure 7: Parallelization steps of the $\mathrm{FE}^{2}$ code.

\section{Applications and Capabilities of the $\mathrm{FE}^{2}$ framework}

In order to validate the two-scale computational approach within the framework of 3D nonlinear composite structures, two test cases have been addressed: the first one is a periodic multilayer composite structure with non-linear, elastoplastic phases. It has been demonstrated that the 
use of an incremental linearized temporal integration approach, there exist a semi-analytical solution for this problem [40]. This test case is utilized as a validation of the implementation of the $\mathrm{FE}^{2}$ framework. The second one is the simulation of three-dimensional composite structure, with a two-phase microstructure: A matrix phase that exhibits a coupled elastoviscoplastic with ductile damage response, reinforced by short glass fibres. The results of such multi-scale simulation are compared with a legacy modelling approach, i.e. the use of an incremental Mori-Tanaka scheme [22].

\subsection{Comparison with semi-analytical homogenization method for elastoplastic multilayer com-} posites

The multi-scale structure simulated is presented in Fig. 8 and is composed at the microscopic scale of a periodic stack of two different layers, one with an elastic response (superscript $e$ ) and the second one with an elastic-plastic response (superscript $p$ ). The volume fraction of the two phases is equal, i.e. $c^{e}=c^{p}=0.5$. The macroscopic shape of the structure is a cuboid. For the elastic-plastic phase, the plastic yield criterion is given by:

$$
f_{p}(\sigma, p)=\operatorname{eq}(\sigma)-R(p)-R_{0} \leq 0
$$

where $\operatorname{eq}(\sigma)$ is the equivalent Von Mises stress and $R_{0}$ is the yield threshold. The hardening function $R(p)$ is chosen under the form of a power law [33]:

$$
R(p)=K \cdot p^{n}
$$

where $K$ and $n$ are material parameters. $p$ is the accumulated plastic strain. The material parameters of the two phases are given in Tab. 3. As discussed in the Section 3.1, periodic boundary conditions are applied to the unit cell of the multilayer material. The macroscopic boundary conditions imposed correspond to a pure shear loading and are such that the relationship between the displacement at the boundary is $\boldsymbol{u}^{0}=\boldsymbol{\varepsilon}^{0} \boldsymbol{n}, \boldsymbol{n}$ being the outward normal of the surface and all the components of the tensor $\varepsilon^{0}$ are zero except $\varepsilon_{12}=\varepsilon_{21}$, see Fig. 9-b. Note that under such conditions, the numerical results of the two Finite Element Analyses should be mesh-independent, since homogeneous fields are considered in all the phases. The results of the two approaches, 
the $\mathrm{FE}^{2}$ and the semi-analytical are identical (Fig. 10), which demonstrates the capability of the computational method to predict the response of 3D non-linear multi-scale composite structures.

Table 3: Material parameters for the two phases

\begin{tabular}{ccc}
\hline & Elastic-plastic phase & \\
\hline Parameter & value & unit \\
\hline$E^{p}$ & 2000 & $\mathrm{MPa}$ \\
$v^{p}$ & 0.3 & - \\
$R_{0}$ & 10 & $\mathrm{MPa}$ \\
$K$ & 60.0 & $\mathrm{MPa}$ \\
$n$ & 0.15 & - \\
\hline \hline & Elastic phase & \\
\hline$E^{e}$ & 6000 & $\mathrm{MPa}$ \\
$v^{e}$ & 0.2 & - \\
\hline
\end{tabular}

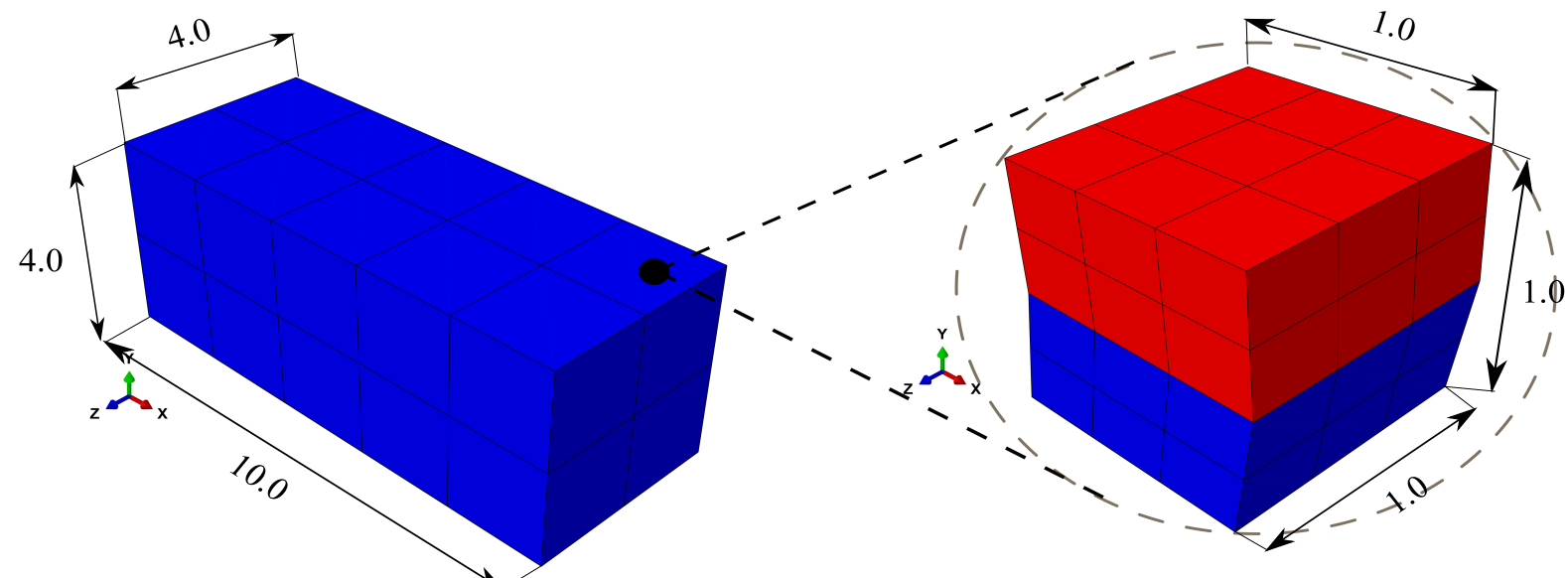

(a) Macroscopic structure

(b) Unit cell

Figure 8: Multilayer composite structure with their microstructure associated with each macroscopic integration point. 


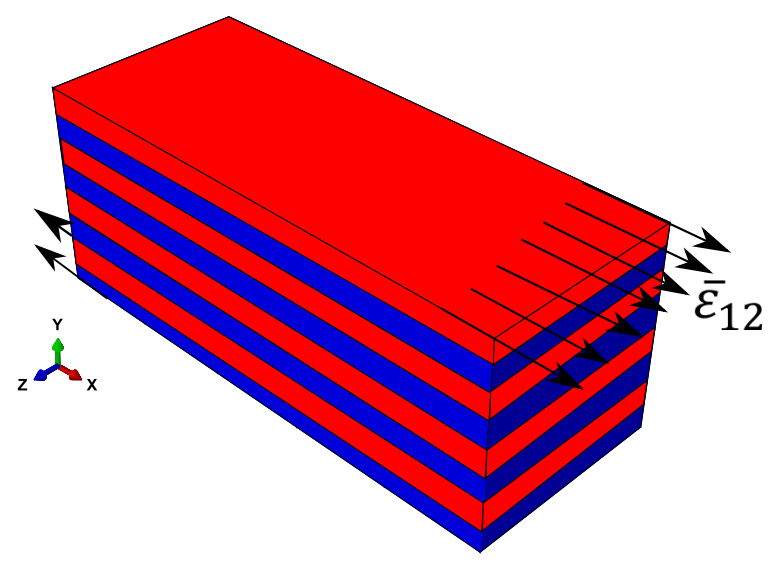

(a) 3D Multilayer composite structure.

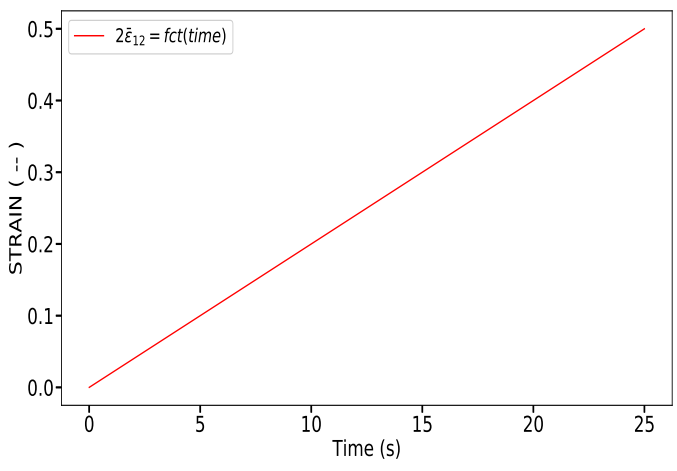

(b) Applied loading path.

Figure 9: Multilayer composite structure under shear loading path.

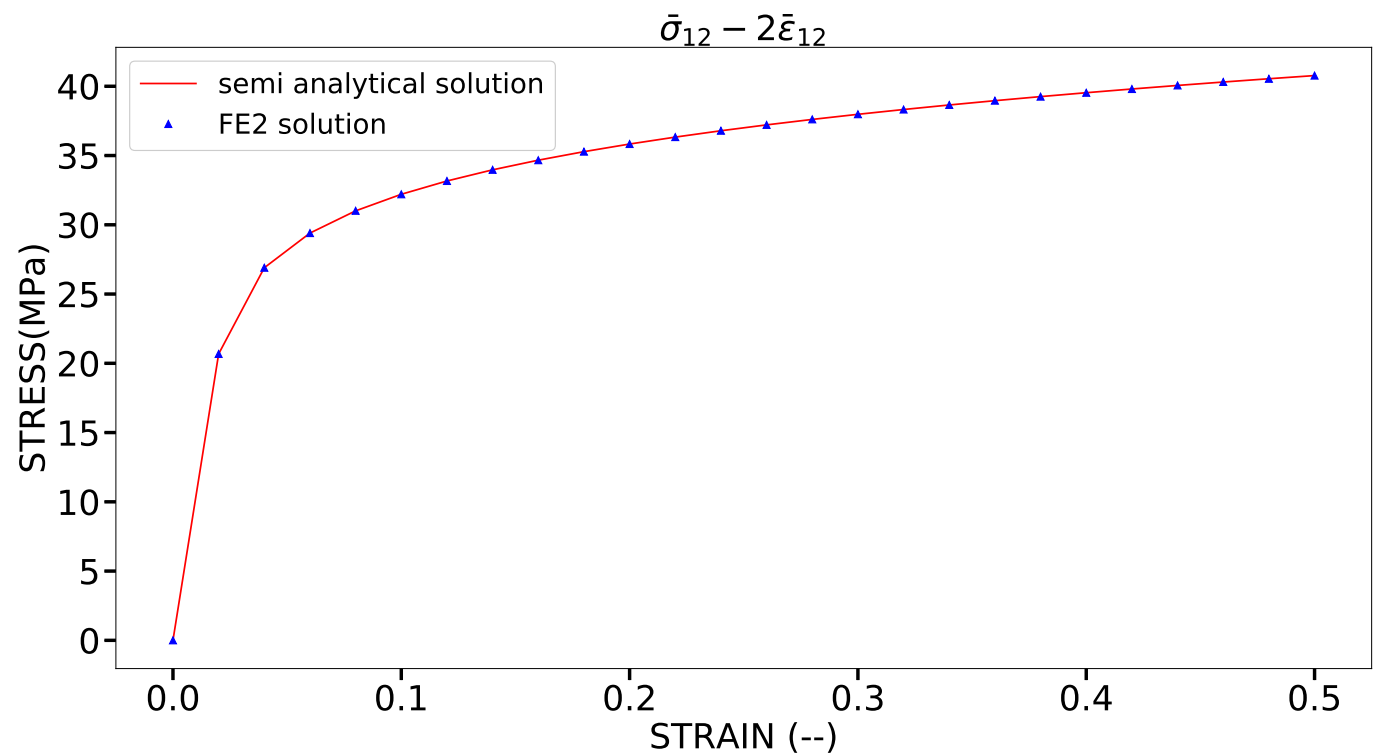

Figure 10: Comparison of the numerical result of $\mathrm{FE}^{2}$ approach with semi-analytical solution on multilayer witch elastoplastic phases in term of macroscopic stress-strain response.

\subsection{D structure (Meuwissen) with short fibre reinforced composite}

To demonstrate the capabilities of the $\mathrm{FE}^{2}$ approach to identify the overall behavior of 3D composite structures close to parts that are commonly manufactured, the second test case is performed on structure where heterogeneous strain and stress fields are observed during a tensile load field. the composite material is considered as an elastoviscoplastic polymer matrix with ductile damage, 
reinforced by aligned glass short fibres arranged in a periodic hexagonal array (Fig. 11). The volume fractions of the matrix and the fibres are $V^{m}=0.925$ and $V^{f}=0.075$ respectively, while the aspect ratio for the elliptic fibre is $(4,1,1)$. The fibres elastic properties are the following: a Young's modulus $E^{f}=72000 \mathrm{MPa}$ and a Poisson's ratio $v^{f}=0.26$. The material properties of the matrix phase are listed in Tab. 4. It should be mentioned that these material parameters are motivated by the work of [11], but they do not consider the viscoelastic response, which is taken into account in that article. Thus, the material properties are related to viscoplastic behavior coupled to damage in polymeric media.

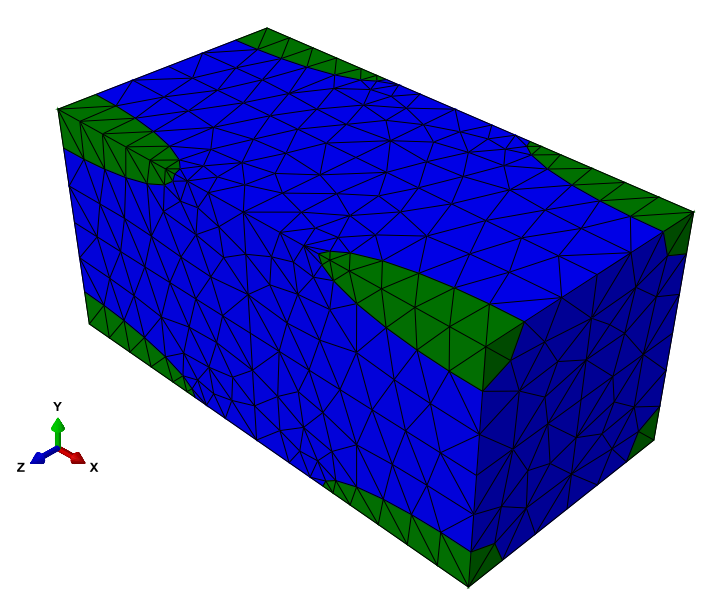

(a) Mesh of the entire unit cell.

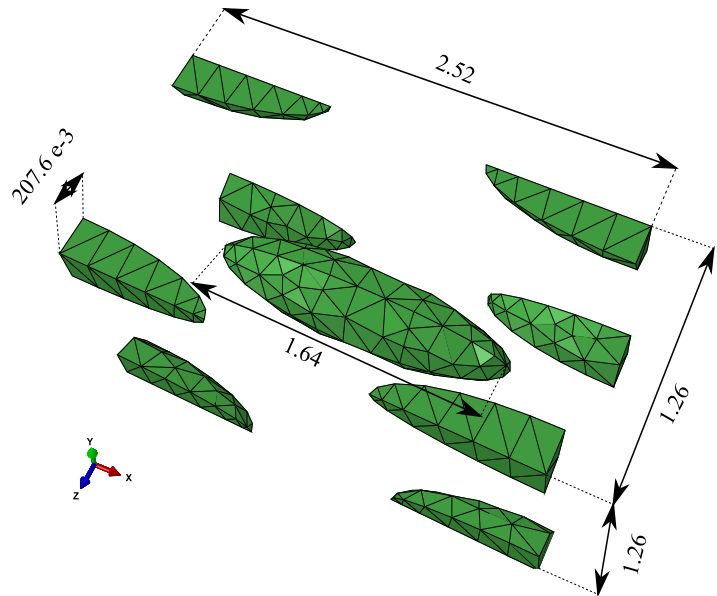

(b) Short fibres reinforcement.

Figure 11: Composite microstructure.

The structure presented in the Fig. 12 - a is clamped at the left side and subjected to the loading path of Fig. 12 b at the right side. The displacement controlled path consists in three loading steps with different velocities $\left(\dot{u}_{x}^{(1)}=1 \mathrm{~mm} . \mathrm{s}^{-1}, \dot{u}_{x}^{(2)}=0.2 \mathrm{~mm} . \mathrm{s}^{-1}, \dot{u}_{x}^{(3)}=0.8 \mathrm{~mm} . \mathrm{s}^{-1}\right)$ followed by an unloading stage at a displacement rate of $\left(\dot{u}_{x}^{(4)}=2 \mathrm{~mm} \cdot \mathrm{s}^{-1}\right)$. The results of the full-field $\mathrm{FE}^{2}$ method are compared with those obtained by using the incremental mean-field Mori-Tanaka method. This method has been widely utilized for the simulation of composites [13] as well as smart structures [41]. Such homogenization scheme is however considered valid under specific cases [22], and some specific corrections might be required [23, 42]. Since the proposed corrections are not unique and depends on the type of composites, the regular incremental method is employed, 
Table 4: Material parameters for polymer matrix

\begin{tabular}{ccc}
\hline Parameter & value & unit \\
\hline \hline$E^{m}$ & 1680 & $\mathrm{MPa}$ \\
$v^{m}$ & 0.3 & - \\
$R_{0}$ & 10 & $\mathrm{MPa}$ \\
$K$ & 365.0 & $\mathrm{MPa}$ \\
$n$ & 0.39 & - \\
$H$ & 180.0 & $\mathrm{MPa} . \mathrm{s}$ \\
$m$ & 0.3 & - \\
$S$ & 6.0 & $\mathrm{MPa}$ \\
$\beta$ & -1.70 & - \\
\hline
\end{tabular}

where the linearized problem is written in term of the anisotropic algorithmic tangent modulus of the non-linear phases [22]. The advantage of the Mori-Tanaka scheme relies in its computational efficiency, since it is a semi-analytical method and accounts for the material non-linearities only on an average sense and not at every local microscopic point in the unit cell. The overall load-displacement response computed using $\mathrm{FE}^{2}$ approach is shown in Fig. 13 and compared to the global response predicted by the mean-field Mori-Tanaka approach. As expected the both approaches predict comparable responses notably in the elastic regime. However, for the viscoplastic regime, the mean-field based simulation does not capture well the strain rate change of the applied loading path and provides stiffer response than the full-field $\mathrm{FE}^{2}$. This aspect is known and occurs when one phase exhibits a non-linear behavior. Similar observations have been reported to the literature especially when the matrix phase behaves as viscoelastic-viscoplastic media [7, 23]. The authors proposed specific numerical formulations to address this limit of mean-field based methods. Fig. 14 demonstrates stress-strain curves at the macroscopic point A (Fig. 15-a). Due 
to the semi-analytical form of the Mori-Tanaka method, the computations are faster than in the $\mathrm{FE}^{2}$ method but it requires a smaller time increment. The results indicate that the response of the two approaches describe the changing of the rate loading caused by the viscous behavior of polymer matrix, but it is clearly shown that the Mori-Tanaka response misdescribed this phenomena because it is more rigid with a considerable loss of plasticity as expected, compared to the $\mathrm{FE}^{2}$. The results illustrate that the response of the composite is highly influenced by the presence of the matrix, exhibiting both viscoplastic response through relaxation phenomena, as well as stiffness reduction during unloading due to the ductile damage.

It is worth noticing that the inelastic characteristics of the different phases are mainly taken into account in the microscale and, accordingly, the unit cell is adequately meshed (6857 elements). The authors have performed several analyses at different meshes of the macroscopic structure and have confirmed that the chosen meshing of 100 elements was sufficient for the purposes of the manuscript.

At a characteristic critical point of the structure (centre of one notch), the deformed macro-scale structure and the microscopic stress response (component 11) of the unit cell that represent a macroscopic integration point $\mathrm{A}$ are shown in Fig. 15. It is clear that at such critical material point, the adopted incremental Mori-Tanaka scheme do not predict the local response with a sufficient accuracy to be able to utilize such results for the computation of damage evolution of fatigue life predictions, which are unavoidable in the case of most load-bearing application of composite structures. Even if the mesh convergence is difficult to reach to obtain exact results, the $\mathrm{FE}^{2}$ framework could provide a standard of predictability that is much higher than the mean-fields methods when a composite is simulated, where the matrix present a strongly non-linear response. It deserves to be mentioned that the periodic homogenization gives excellent results for 3D structures, and the numerical accuracy depends on that of the FE calculations. However, when addressing plate or shell structures, the periodic homogenization requires proper modifications, as described in [43], due to the loss of periodicity in the thickness direction (out of plane). This results in less accurate prediction for the out of plane Poisson ratio. Nevertheless, the out of plane periodicity can be reasonably assumed when the microstructure contains high number of fibers or layers in the thickness direction. 


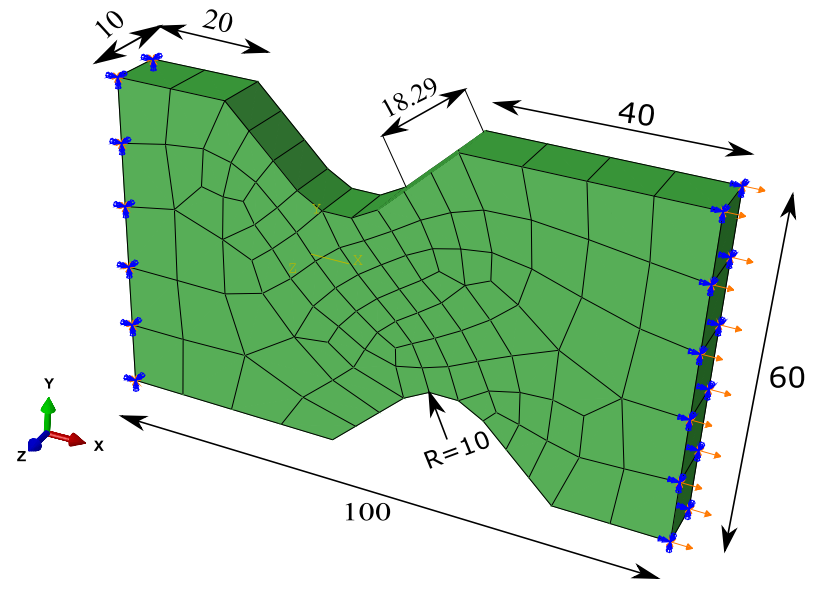

(a) Mesh of the entire 3D composite structure.

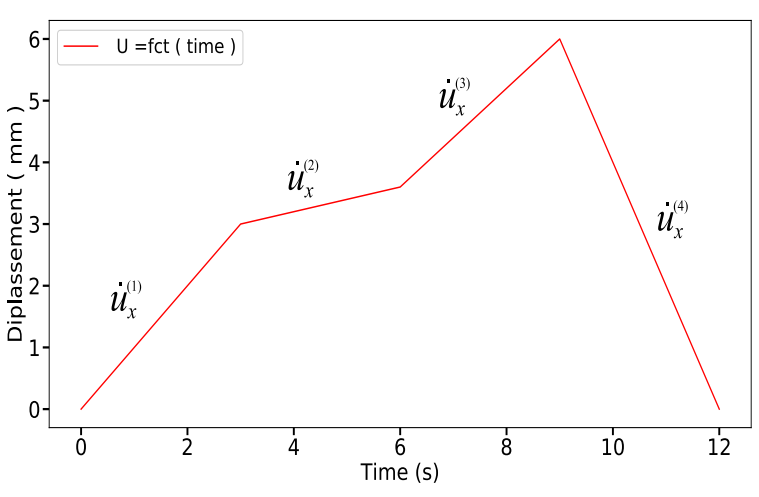

(b) Applied loading path.

Figure 12: Tensile and compression test on the 3D Meuwissen test tube.

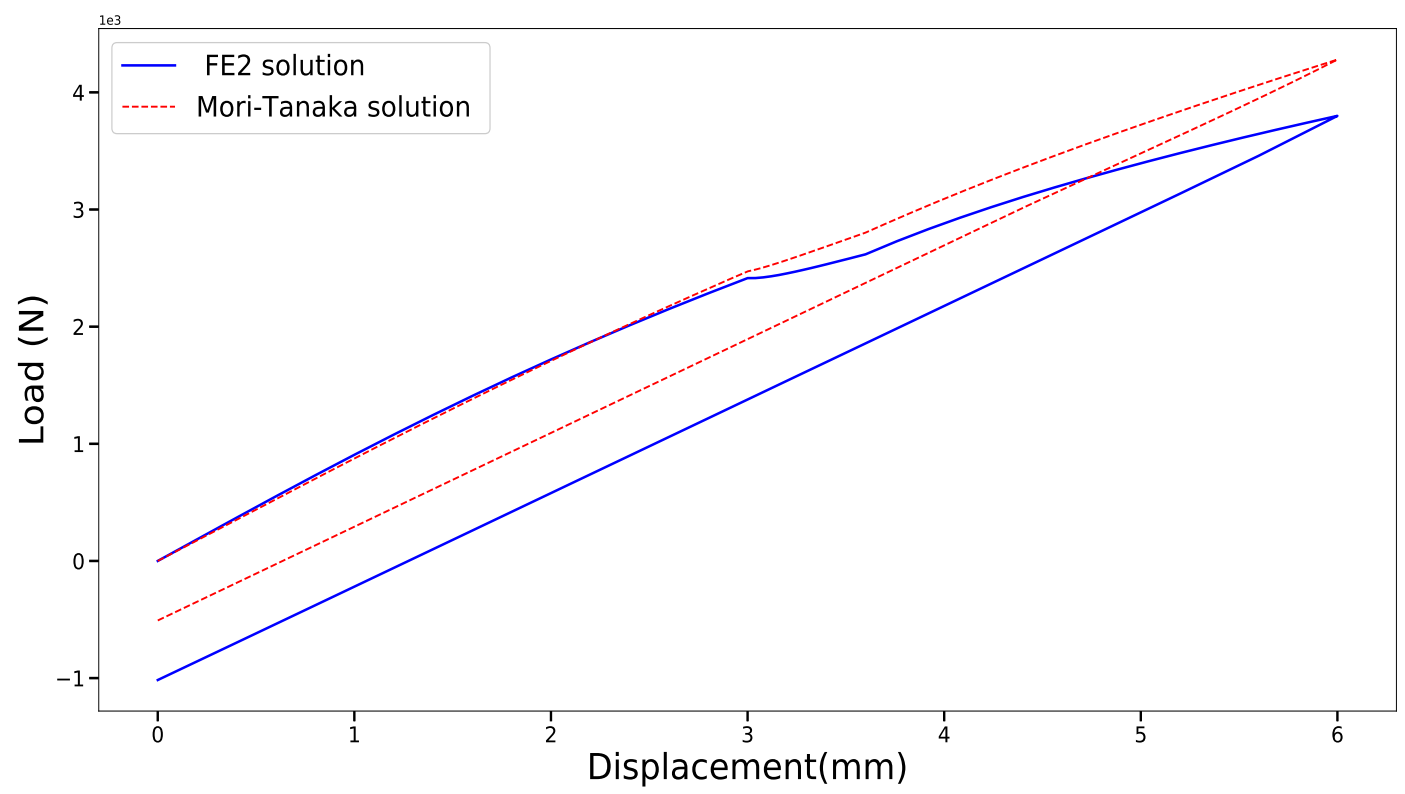

Figure 13: The overall load-displacement response of the structure in the directions 11. Comparison between the $\mathrm{FE}^{2}$ and the Mori-Tanaka solution. 

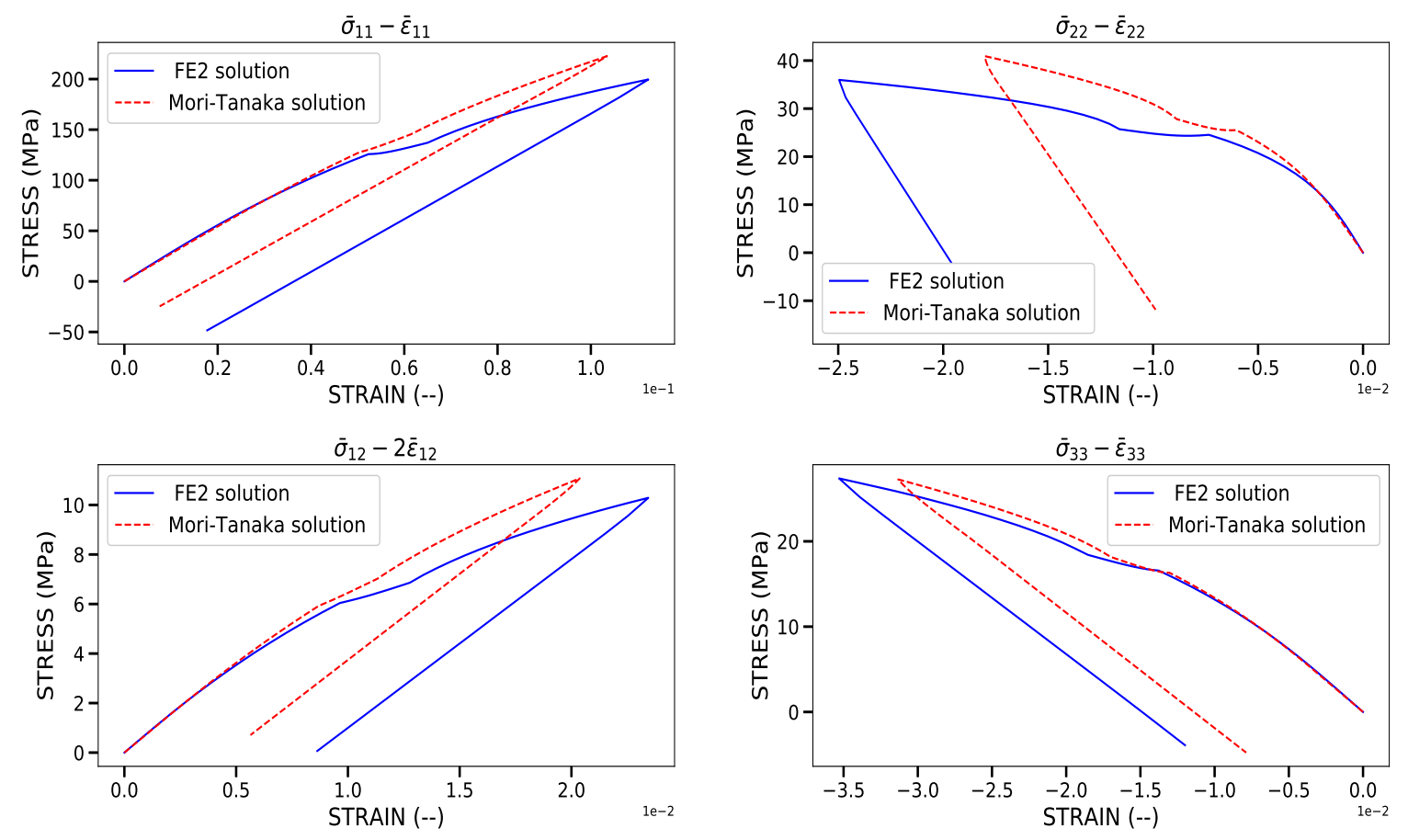

Figure 14: Macroscopic response of the composite at point A in term of stress-strain in the directions 11, 22, 33 and shear 12 (comparison between the $\mathrm{FE}^{2}$ and the Mori-Tanaka solution).

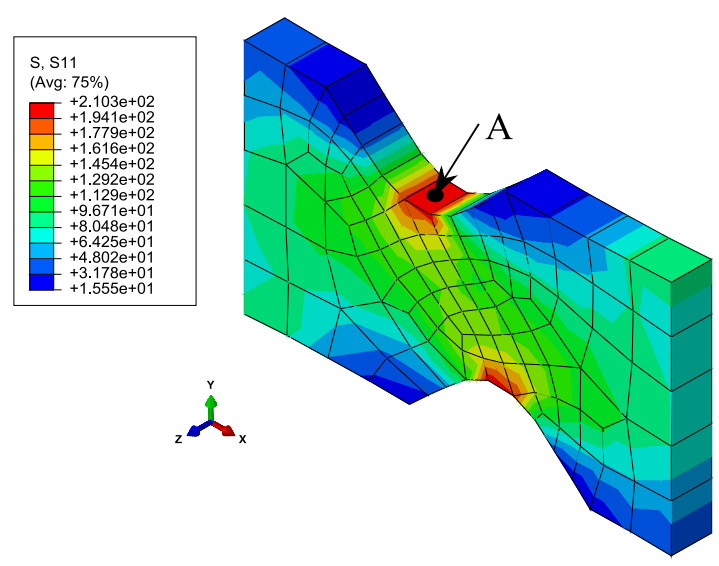

(a) Macroscopic stress field of the composite structure.

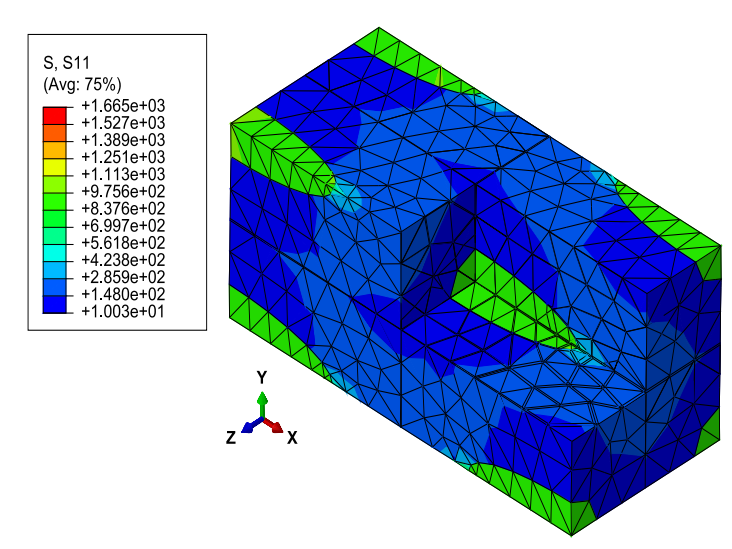

(b) Microscopic stress field of the microstructure at point A.

Figure 15: $\mathrm{FE}^{2}$ solution with ABAQUS/Standard (component 11). 


\subsection{Complex $3 D$ structure with corner shape}

In this section, a second 3D composite structure is simulated in order to illustrate the capability and the flexibility of the approach, when more complex boundary conditions are applied to the macroscopic structure. The modelled structure consists of a 3D part having a corner shape (Fig. 16-a). It is made of a thermoplastic aligned short fibre reinforced composite in which the matrix and reinforcement phases exhibit the same behavior as in Section 4.2. The structure is clamped at the bottom side and subjected to a normal uniform displacement path at left side (Fig. 16-b). The displacement controlled path consists in two loading steps with different displacement rates $\left(\dot{u}_{x}^{(1)}=2.1875 \mathrm{~mm} \cdot \mathrm{s}^{-1}, \dot{u}_{x}^{(2)}=0.15625 \mathrm{~mm} \cdot \mathrm{s}^{-1}\right)$ and an unloading step at a displacement rate of $\left(\dot{u}_{x}^{(3)}=0.9375 \mathrm{~mm} \cdot \mathrm{s}^{-1}\right)$.

In Figs. 17 and 18 , the whole response of the composite in terms of macroscopic stress vs strain are depicted at two distinct points A and B (Fig. 19.-c). The approach is able to reproduce the effect of such microstructure on the overall response of the composite, as on the most stressed point shown in Fig. 19. Indeed, on the clamped part (point A), it is clear that the structure is subjected to a tensile load according to the 22 direction, and a shear load in the direction 12 . These results are attributable primarily by the macroscopic boundary conditions. Furthermore, for the point B, a high stress value in the direction of loading was noticed. Fig. 19. c shows the stress response (component 11) of the macroscopic structure and the resulting microscopic stress in the two unit cells situated at two different macroscopic integration points A and B (Figs. 19. a and 19.b respectively). The response of the composite is highly affected by the matrix behavior through the relaxation phenomena caused by the change of the loading rate. The apparent stiffness reduction during the unloading caused by the ductile damage in the matrix is clearly observed.

For the parallelization procedure and with the same number of increments (42 increments), the computation becomes 18 times faster than the non parallel solution. The actual computational time of the analysis performed on 18 processors was approximately $72 \mathrm{~h}$ for a macroscopic structure containing 90 elements of type C3D8 with 6857 microscopic elements of type C3D4. 


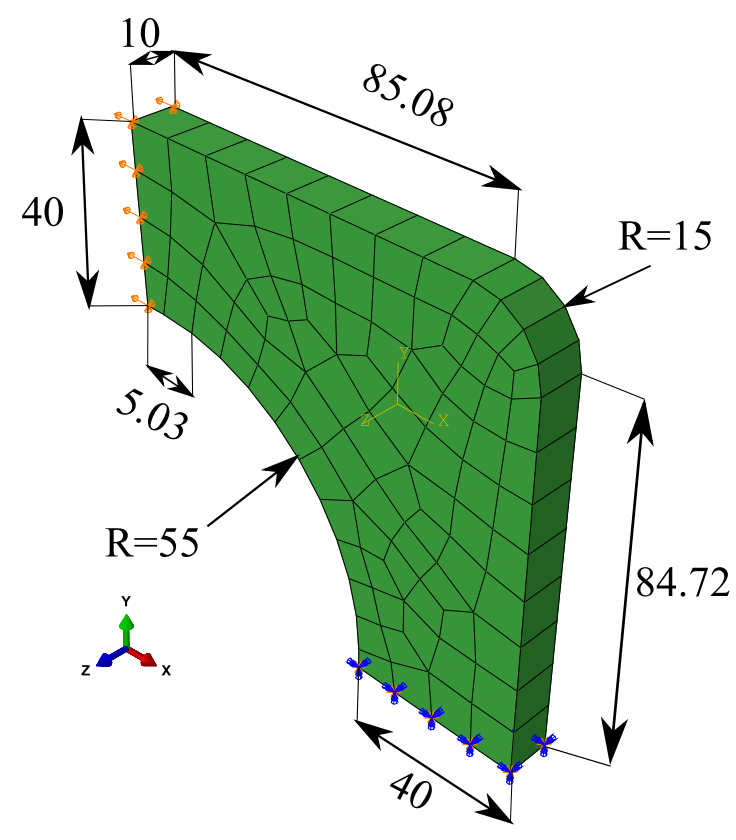

(a) Macroscopic mesh of the 3D composite structure.

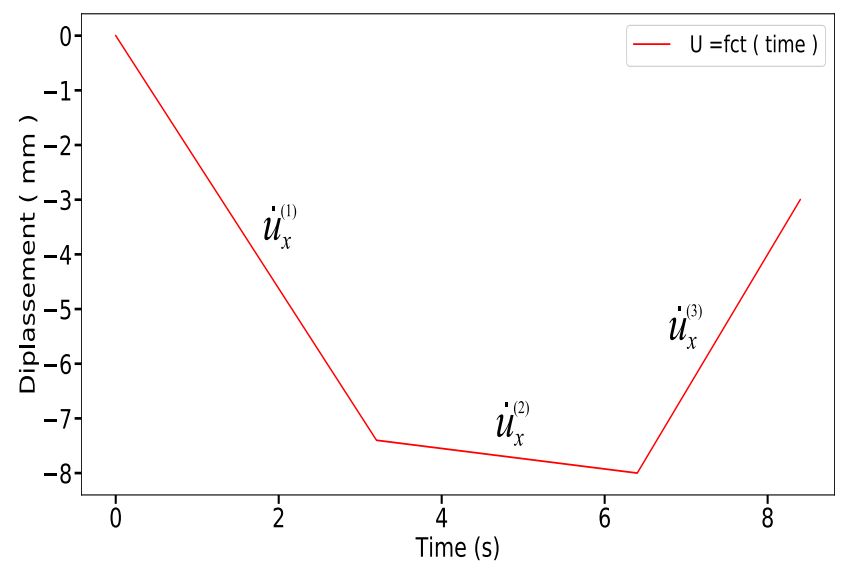

(b) Applied loading path.

Figure 16: Tensile and compression test on the 3D composite structure with corner.
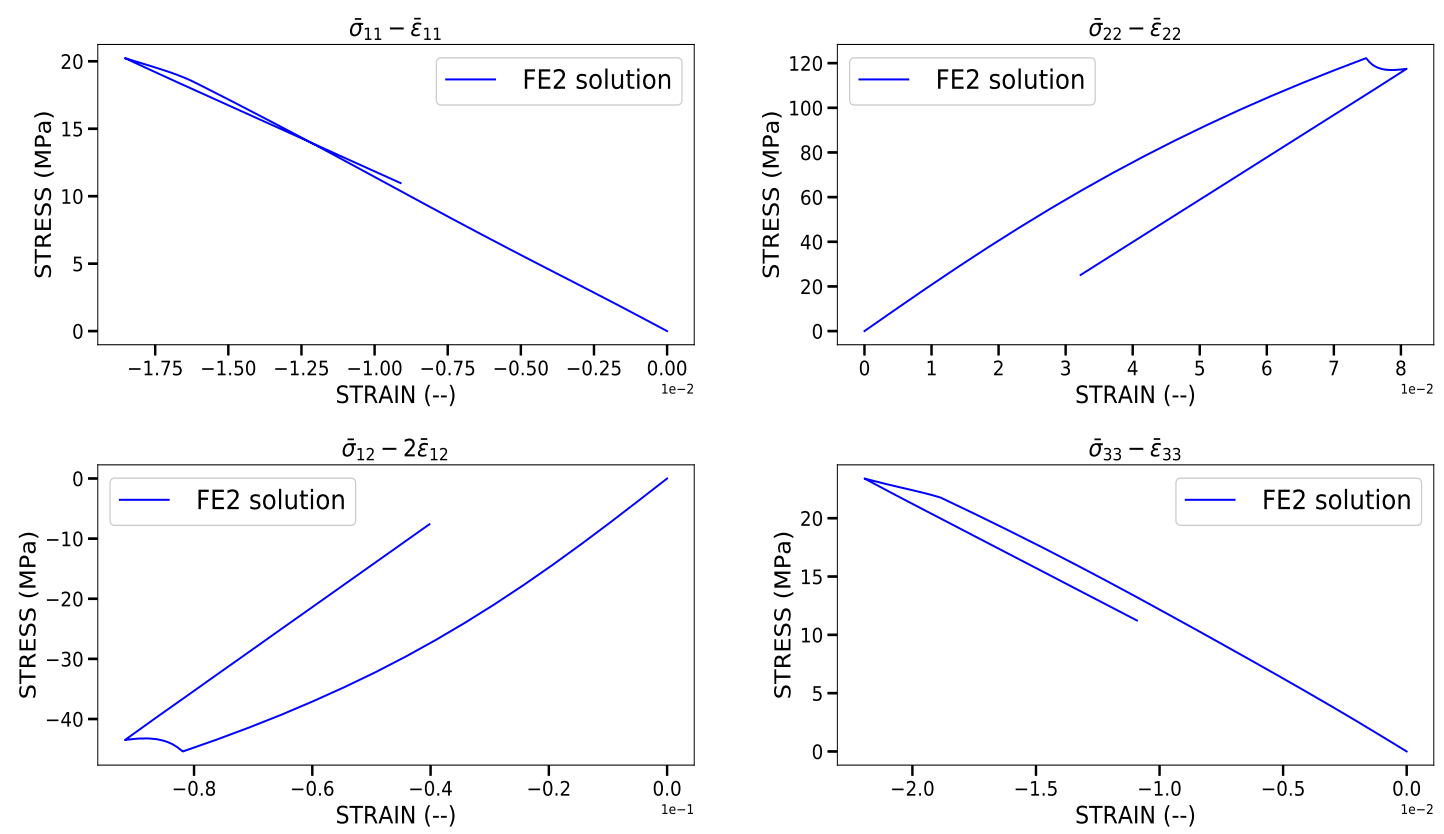

Figure 17: Macroscopic response of the composite at point A in term of stress-strain in the directions 11, 22, 33 and shear 12. 

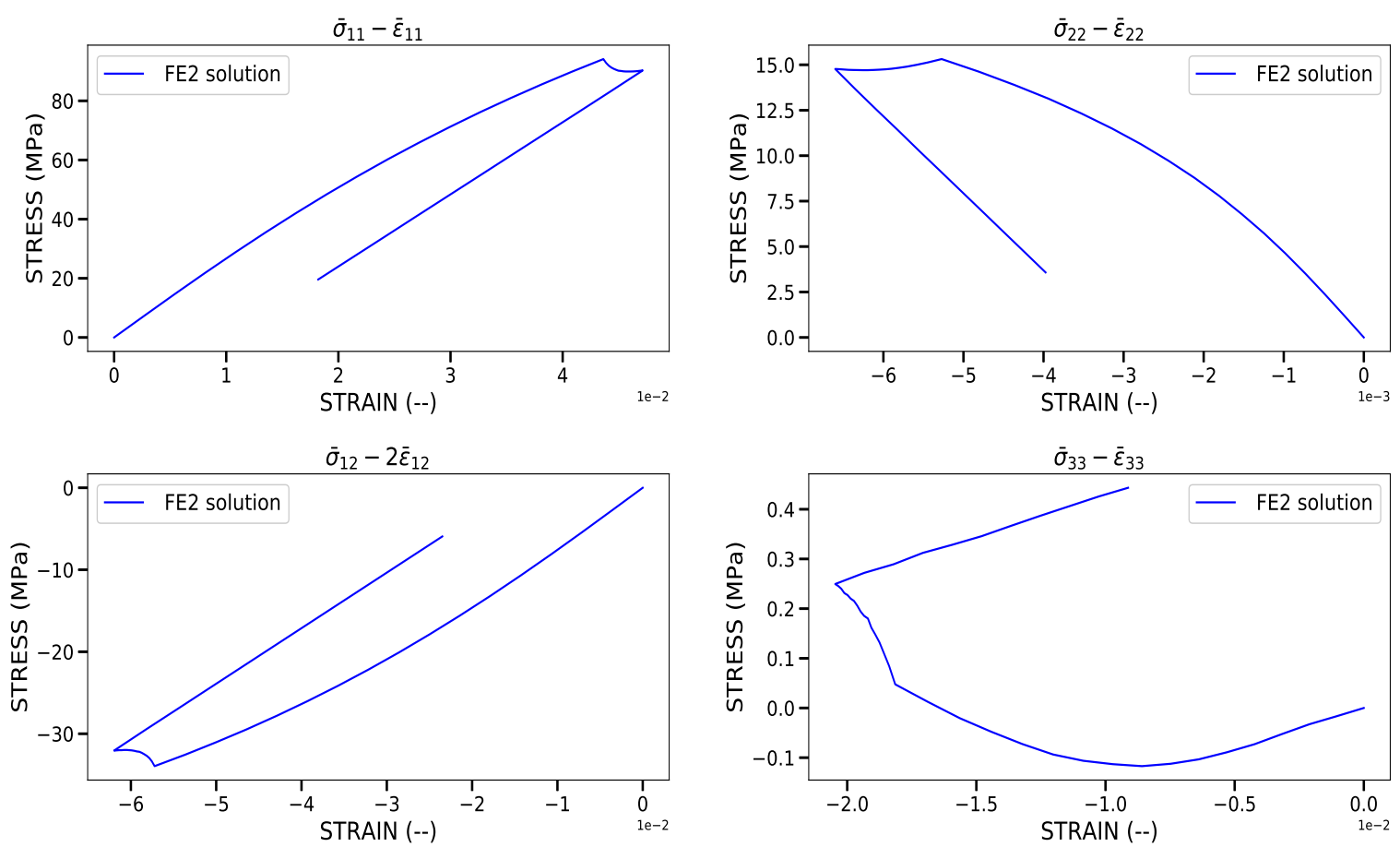

Figure 18: Macroscopic response of the composite at point B in term of stress-strain in the directions 11, 22, 33 and shear 12. 


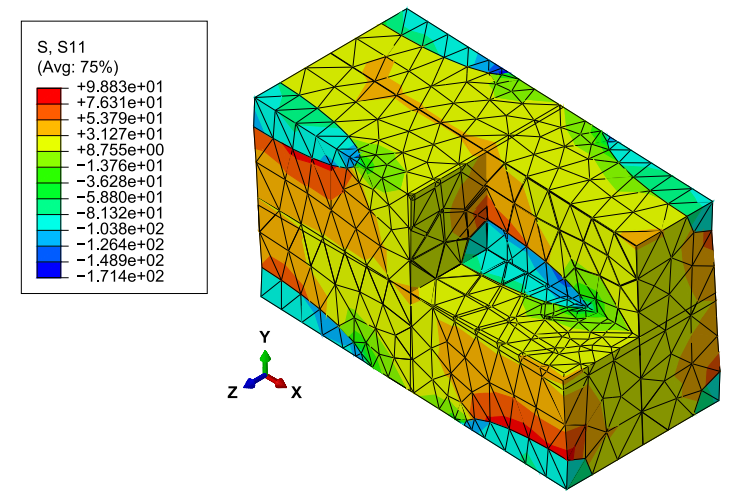

(a) Microscopic stress field of the microstructure at point A.
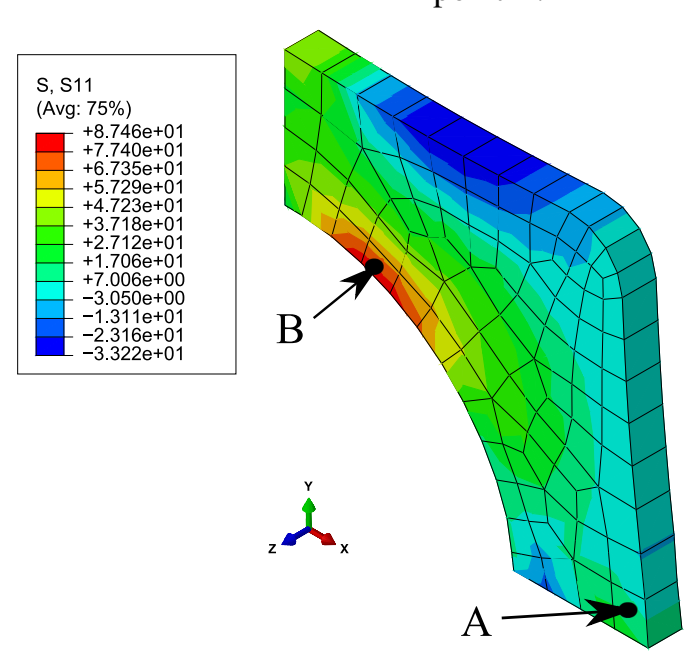

(c) Macroscopic stress field of the 3D composite structure (component 11).

Figure 19: $\mathrm{FE}^{2}$ solution with ABAQUS/Standard (component 11).

\section{Conclusions and further work}

This work presents a non-linear three-dimensional two-scale finite element $\left(\mathrm{FE}^{2}\right)$ framework fully integrated in the Finite Element Analysis Package ABAQUS/Standard, using parallel computation. The main advantage of the method is that it does not require an analytical form for the constitutive law at the macro-scale, while accounting for the microstructural effects and the local behaviors. It can integrate any kind of periodic microstucture with any type of non-linear behavior of the reinforcement (fibres and/or particles) and the matrix (plastic, viscoelastic, viscoplastic and 
damage).

The multi-scale strategy has been tested on three independent numerical examples: In the first example, a 3D multilayer composite structure with elastoplastic phases is simulated and compared with semi analytical solution, to validate the numerical implementation. In the second example, a short glass fibre reinforced composite with elastoviscoplastic-damageable matrix under complex loading is examined through the $\mathrm{FE}^{2}$ strategy and the results are compared to those obtained by the Mori-Tanaka method. The obtained responses were in agreement with those presented in the literature in similar cases, and highlight the importance of utilizing full-field method for a generic modelling strategy with high predictability capabilities. In the third example, 3D a complex composite structure with corner shape is simulated in which the microstucture is made of an elastoviscoplastic matrix with ductile damage reinforced by short glass fibre. The capabilities of such approach to reproduce the effect of such microstructure on the macrostructure response at each macroscopic integration point has been demonstrated. The response of the structure clearly highlights creep and relaxation phenomena, which are characteristic for rate dependent responses. This viscous behavior and the stiffness reduction observed during unloading have been induced by the viscoplastic nature of the polymer matrix. It worth noticing that for composites where the matrix is viscoplastic material, the Mori-Tanaka method under proper modifications can provide quite accurate results [44, 7] compared to the full-field based approach.

A last advantage of this approach is that it can be extended to predict the overall fully coupled thermomechanical response of 3D composite structures [45, 40] with more complex mechanisms between fibres-matrix as interfacial damage mechanisms. Such fully-coupled analyses on multiscale structures should be of a high interest for industrial applications that are usually computed with commercial finite element analyses packages. 
[1] Moreau S, Chrysochoos A, Muracciole J-M, and Wattrisse B. Analysis of thermoelastic effects accompanying the deformation of pmma and pc polymers. Comptes Rendus Mécanique, 333(8):648 - 653, 2005.

[2] Akhtar S-k, Pamies O-L, and Kazmi R. Thermo-mechanical large deformation response and constitutive modeling of viscoelastic polymers over a wide range of strain rates and temperatures. International Journal of Plasticity, 22(4):581 - 601, 2006.

[3] Duan Y, Saigal A, Greif R, and Zimmerman M-A. A uniform phenomenological constitutive model for glassy and semi crystalline polymers. . Polymer engineering and science, 41:1322-1328, 2001.

[4] Achour N, Chatzigeorgiou G, Meraghni F, Chemisky Y, and Fitoussi J. Implicit implementation and consistent tangent modulus of a viscoplastic model for polymers. International Journal of Mechanical Sciences, 103:297 $-305,2015$.

[5] Drozdov A.D and Christiansen J-deC. Cyclic viscoplasticity of solid polymers: The effects of strain rate and amplitude of deformation. Polymer, 48(10):3003 - 3012, 2007.

[6] Miled B, Doghri I, and Delannay L. Coupled viscoelastic-viscoplastic modeling of homogeneous and isotropic polymers: Numerical algorithm and analytical solutions. Computer Methods in Applied Mechanics and Engineering, 200(47):3381 - 3394, 2011.

[7] Miled B, Doghri I, Brassart L, and Delannay L. Micromechanical modeling of coupled viscoelastic-viscoplastic composites based on an incrementally affine formulation. International Journal of Solids and Structures, 50(10): 1755 - 1769, 2013

[8] Launay A, Maitournam M-H, Marco Y, Raoult I, and Szmytka F. Cyclic behaviour of short glass fibre reinforced polyamide: Experimental study and constitutive equations. International Journal of Plasticity, 27(8):1267 $1293,2011$.

[9] Launay A, Maitournam M-H, Marco Y, and Raoult I. Multiaxial fatigue models for short glass fiber reinforced polyamide - part i: Nonlinear anisotropic constitutive behavior for cyclic response. International Journal of Fatigue, 47:382 - 389, 2013.

[10] Krairi A and Doghri I. A thermodynamically-based constitutive model for thermoplastic polymers coupling viscoelasticity, viscoplasticity and ductile damage. International Journal of Plasticity, 60:163 - 181, 2014.

[11] Praud F, Chatzigeorgiou G, Bikard J, and Meraghni F. Phenomenological multi-mechanisms constitutive modelling for thermoplastic polymers, implicit implementation and experimental validation. Mechanics of Materials, $114: 9-29,2017$.

[12] Mori T and Tanaka K. Average stress in matrix and average elastic energy of materials with misfitting inclusions. Acta Metallurgica, 21(5):571 - 574, 1973.

[13] Doghri I and Ouaar A. Homogenization of two-phase elasto-plastic composite materials and structures: Study of tangent operators, cyclic plasticity and numerical algorithms. International Journal of Solids and Structures, 40(7): $1681-1712,2003$. 
[14] Hill R. A self-consistent mechanics of composite materials. Journal of the Mechanics and Physics of Solids, 13(4):213 - 222, 1965.

[15] Budiansky B. Micromechanics. Computers and Structures, 16(1):3 - 12, 1983.

[16] Walpole L-J. On bounds for the overall elastic moduli of inhomogeneous systems-i. Journal of the Mechanics and Physics of Solids, 14(3):151 - 162, 1966.

[17] Graeme W. Milton and Robert V. Kohn. Variational bounds on the effective moduli of anisotropic composites. Journal of the Mechanics and Physics of Solids, 36(6):597 - 629, 1988.

[18] Castañeda P-P. The effective mechanical properties of nonlinear isotropic composites. Journal of the Mechanics and Physics of Solids, 39(1):45 - 71, 1991.

[19] Meraghni F and Benzeggagh M-L. Micromechanical modelling of matrix degradation in randomly oriented discontinuous-fibre composites. Composites Science and Technology, 55(2):171 - 186, 1995.

[20] Meraghni F, Desrumaux F, and Benzeggagh M-L. Implementation of a constitutive micromechanical model for damage analysis in glass mat reinforced composite structures. Composites Science and Technology, 62(16):2087 $-2097,2002$.

[21] Gavazzi A-C and Lagoudas D-C. On the numerical evaluation of eshelby's tensor and its application to elastoplastic fibrous composites. Computational Mechanics, 7(1):13-19, 1990. cited By 118.

[22] Lagoudas D-C, Gavazzi A-C, and Nigam H. Elastoplastic behavior of metal matrix composites based on incremental plasticity and the mori-tanaka averaging scheme. Computational Mechanics, 8(3):193-203, 1991. cited By 76.

[23] Chaboche J-L, Kanouté P, and Roos A. On the capabilities of mean-field approaches for the description of plasticity in metal matrix composites. International Journal of Plasticity, 21(7):1409 - 1434, 2005.

[24] Frédéric F. Multiscale fe2 elastoviscoplastic analysis of composite structures. Computational Materials Science, 16(1):344-354, 1999.

[25] Feyel F and Chaboche J-L. Fe2 multiscale approach for modelling the elastoviscoplastic behaviour of long fibre sic/ti composite materials. Computer Methods in Applied Mechanics and Engineering, 183(3):309 - 330, 2000.

[26] Nezamabadi S, Yvonnet J, Zahrouni H, and Potier-Ferry M. A multilevel computational strategy for handling microscopic and macroscopic instabilities. Computer Methods in Applied Mechanics and Engineering, 198(27):2099 - 2110, 2009.

[27] Nezamabadi S, Zahrouni H, Yvonnet J, and Potier-Ferry M. A multiscale finite element approach for buckling analysis of elastoplastic long fiber composites. International Journal for Multiscale Computational Engineering, 8:287-301, 2010.

[28] Asada T and Ohno N. Fully implicit formulation of elastoplastic homogenization problem for two-scale analysis. International Journal of Solids and Structures, 44(22):7261 - 7275, 2007.

[29] Tchalla A, Belouettar S, Makradi A, and Zahrouni H. An abaqus toolbox for multiscale finite element compu- 
tation. Composites Part B: Engineering, 52:323 - 333, 2013.

[30] Schröder J, Labusch M, and Keip M-A. Algorithmic two-scale transition for magneto-electro-mechanically coupled problems: Fe2-scheme: Localization and homogenization. Computer Methods in Applied Mechanics and Engineering, 302:253 - 280, 2016.

[31] Papadopoulos V and Tavlaki M. The impact of interfacial properties on the macroscopic performance of carbon nanotube composites. a fe2-based multiscale study. Composite Structures, 136:582 - 592, 2016.

[32] Praud F, Chatzigeorgiou G, Chemisky Y, and Meraghni F. Modélisation multi-échelle des composites tissés à matrice thermoplastique sous chargements cycliques non proportionnels. JNC20 : Journées Nationales sur les Composites 2017, Jun 2017, Champs-sur-Marne, France. JNC 20: <hal-01576736>, 2017.

[33] Lemaitre J and Chaboche J-L. Mechanics of solid materials. Cambridge University Press, 1990.

[34] Krairi A and Doghri I. A thermodynamically-based constitutive model for thermoplastic polymers coupling viscoelasticity, viscoplasticity and ductile damage. International Journal of Plasticity, 60:163 - 181, 2014.

[35] Lemaitre J. Coupled elasto-plasticity and damage constitutive equations. Computer Methods in Applied Mechanics and Engineering, 51(1):31 - 49, 1985.

[36] Li S. On the unit cell for micromechanical analysis of fibre-reinforced composites. Proceedings of the Royal Society of London A, 455(1983):815-838, 1999.

[37] Li S. General unit cells for micromechanical analyses of unidirectional composites. Composites: Part A, $32: 815-826,2000$.

[38] Shuguang L and Anchana W. Unit cells for micromechanical analyses of particle-reinforced composites. Mechanics of Materials, 36(7):543 - 572, 2004.

[39] Simo J-C and Hughes T-J-R. Computational Inelasticity. Springer-Verlag, New York, 1998.

[40] Chatzigeorgiou G, Chemisky Y, and Meraghni F. Computational micro to macro transitions for shape memory alloy composites using periodic homogenization. Smart Materials and Structures, 24(3):035009, 2015.

[41] Piotrowski B, Ben Zineb T, Patoor E, and Eberhardt A. Modeling of niobium precipitates effect on the ni47ti44nb9 shape memory alloy behavior. International Journal of Plasticity, 36:130 - 147, 2012.

[42] Brassart L, Doghri I, and Delannay L. Homogenization of elasto-plastic composites coupled with a nonlinear finite element analysis of the equivalent inclusion problem. International Journal of Solids and Structures, 47(5):716 - 729, 2010.

[43] Kalamkarov A-L and Kolpakov A-G. Analysis, design and optimization of composite structures. Wiley, West Sussex, 1997.

[44] Mercier S and Molinari A. Homogenization of elastic-viscoplastic heterogeneous materials: Self-consistent and mori-tanaka schemes. International Journal of Plasticity, 25(6):1024 - 1048, 2009.

[45] Berthelsen R, Denzer R, Oppermann P, and Menzel A. Computational homogenisation for thermoviscoplasticity: application to thermally sprayed coatings. Comput Mech, 60:739-766, 2017. 
[46] Lemaitre J and Desmorat R. Engineering damage mechanics : ductile, creep, fatigue and brittle failure. Springer, 2005.

[47] Vasiukov D, Panier S, and Hachemi A. Non-linear material modeling of fiber-reinforced polymers based on coupled viscoelasticity-viscoplasticity with anisotropic continuous damage mechanics. Composite Structures, $132: 527-535,2015$.

[48] Suquet P. Analyse limite et homogeneisation. Comptes Rendus de l'Académie des sciences, Paris II, 295:1355-1358, 1983.

[49] Suquet P. Local and global aspects in the mathematical theory of plasticity. Plasticity Today, pages 279-309, 1985. 